\title{
Cascades in the Dynamics of Measured Foliations
}

\section{Citation}

McMullen, Curtis T. Forthcoming. "Cascades in the Dynamics of Measured Foliations." Annales Scientifiques de l'École Normale Supérieure.

\section{Permanent link}

http://nrs.harvard.edu/urn-3:HUL.InstRepos:11891557

\section{Terms of Use}

This article was downloaded from Harvard University's DASH repository, and is made available under the terms and conditions applicable to Open Access Policy Articles, as set forth at http:// nrs.harvard.edu/urn-3:HUL.InstRepos:dash.current.terms-of-use\#OAP

\section{Share Your Story}

The Harvard community has made this article openly available.

Please share how this access benefits you. Submit a story.

Accessibility 


\title{
Cascades in the dynamics of measured foliations
}

\author{
Curtis T. McMullen*
}

17 March 2012

\section{Contents}

1 Introduction . . . . . . . . . . . . . . . . . 1

2 Invariants of foliations . . . . . . . . . . . 6

3 Harmonic foliations on Riemann surfaces . . . . . . . . . . . . 10

4 Recurrence, divergence and flux . . . . . . . . . . . 13

5 Cubic examples . . . . . . . . . . . . . . . 16

6 Foliations of rank two . . . . . . . . . . . . . 22

7 Genus two . . . . . . . . . . . . . . . . 24

8 Bifurcations and self-similarity . . . . . . . . . . 29

9 Coupled rotations . . . . . . . . . . . . . . . . 32

10 Quadratic examples .................. 36

A The periodic foliations of a Teichmüller curve . . . . . . . 39

\section{Introduction}

Let $X$ be a compact Riemann surface of genus $g$. Every harmonic 1-form $\rho$ on $X$ is the pullback of a linear form under a suitable period map

$$
\pi: X \rightarrow E \cong \mathbb{R}^{r} / \mathbb{Z}^{r}
$$

The leaves of the associated measured foliation $\mathcal{F}(\rho)$ of $X$ map under $\pi$ into the leaves of an irrational foliation of $E$.

When $r=2$, the behavior of $\mathcal{F}(\rho)$ is strongly influenced by the degree of the period map. For example, if $\mathcal{F}(\rho)$ is periodic then its degree must be zero. At the other extreme, in $\S 6$ we will see:

Theorem 1.1 There is no minimal foliation of degree zero.

${ }^{*}$ Research supported in part by the NSF and MSRI. 
The degree depends only on the absolute periods of $\rho$, given by the real cohomology class $[\rho] \in H^{1}(X)$. In the case of genus $g=2$, there is one important remaining invariant, the relative period of $\rho$ along a path connecting its zeros (see $\S 7)$. In $\S 8$ we will show:

Theorem 1.2 Let $\left(X_{t}, \rho_{t}\right)$ be a family of harmonic forms of genus two with constant absolute periods and relative period t. Assume $\left(X_{0}, \rho_{0}\right)$ has degree zero. Then the bifurcation locus

$$
B=\left\{t: \mathcal{F}\left(\rho_{t}\right) \text { is not periodic }\right\}
$$

is a closed, countable subset of $\mathbb{R}$ which embeds in $\omega^{\omega}$.

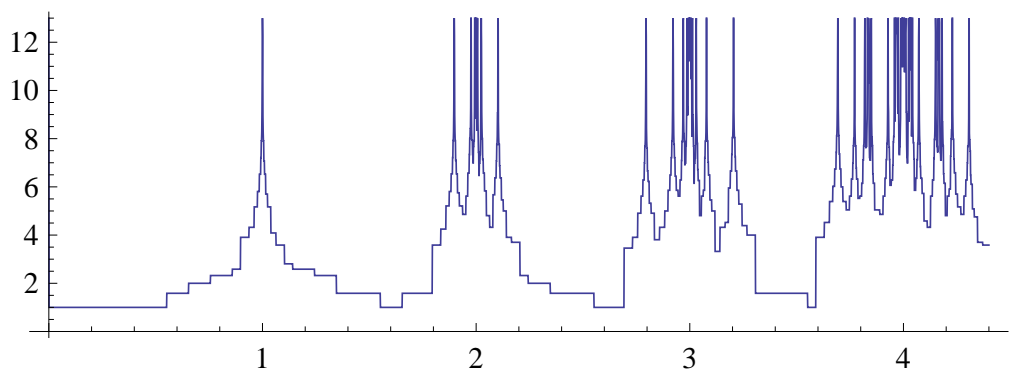

Figure 1. Cascades in the dynamics of $F_{t}:[0,1] \rightarrow[0,1]$.

Example: 1-dimensional dynamics. The spikes in Figure 1 give the bifurcation locus for a family $\left(X_{t}, \rho_{t}\right)$ that depends on an irrational number $L \in(0,1)$. Here a transversal to $\mathcal{F}\left(\rho_{t}\right)$ determines an interval exchange map

$$
F_{t}:[0,1] \rightarrow[0,1]
$$

The map $F_{t}$ rotates $[0,1]$ by $t$, then rotates the subintervals $[0, L]$ and $[L, 1]$ each by $-t$. (Rotation of $[0, a]$ by $b$ is given by $x \mapsto(x+b) \bmod a$.)

The period $N(t)$ of $F_{t}(x)$ is finite iff $t \notin B$. The Figure shows the graph of $y=\log _{2} N(t)$ (calculated using Rauzy induction) in the case $L=\pi / 7$, whose behavior is typical. Theorem 1.2 shows that structural stability is dense in this and similar families of interval exchange transformations.

Homological invariants. To place these results in a broader setting, consider a general closed 1 -form $\rho$ on a compact, oriented $n$-manifold $X$. The Poincaré dual of $[\rho] \in H^{1}(X)$ maps to a natural cycle

$$
\operatorname{flux}(X, \rho) \in H_{n-1}(E),
$$


which records the average distribution of leaves under the period map $\pi$ : $X \rightarrow E$.

The vanishing of flux generalizes the vanishing of degree. As we will see in $\S 4$, zero flux encourages the leaves of $\mathcal{F}(\rho)$ to double back in a manner that is delicate to combine with minimality. Minimal foliations with zero flux in genus three will be discussed in $§ 5$. Such examples cannot exist in genus two, as we will see in $\S 6$ and $\S 7$.

A measurable set $A \subset X$ is saturated if it is a union of leaves of $\mathcal{F}(\rho)$. In this case $d(\rho \mid A)=0$ as a current. We define the content of $\rho$ to be the smallest convex set

$$
C(\rho) \subset H^{1}(X)
$$

containing $[\rho \mid A]$ for every saturated set. In $\S 2$ we show this dynamical invariant is upper semicontinuous, in the sense that

$$
\lim \sup C\left(\rho_{n}\right) \subset C(\rho)
$$

whenever $\rho_{n} \rightarrow \rho$.

Similarly, when $(X, \rho)$ has rank $r=n$, we can make sense of the degree for any saturated set, and we define

$$
\operatorname{deg}^{+}(X, \rho)=\sup _{A} \operatorname{deg}(\pi \mid A) .
$$

In the context of Theorem 1.2, in $\S 8$ we will show:

Theorem 1.3 The quantity $d(t)=\operatorname{deg}^{+}\left(X_{t}, \rho_{t}\right) \geq 0$ is an integer, $B$ is the locus where $d(t)>0$, and we have

$$
\limsup _{s \rightarrow t} d(s) \leq d(t)-1
$$

whenever $t \in B$.

We emphasize that $d(s)$ is bounded by $d(t)-1$ (and not just $d(t)$ ) for all $s$ near $t$. This evaporation of degree under perturbations is one of the main new phenomena we establish in this paper. The properties of $B$ stated in Theorem 1.2 follow from it immediately, and it plays a key role in the analysis of isoperiodic forms [Mc9].

Quadratic fields. In $\S 8$ we will also show:

Theorem 1.4 If the absolute periods of $\rho_{0}$ lie in a real quadratic field, then $B$ is self-similar about every point. 

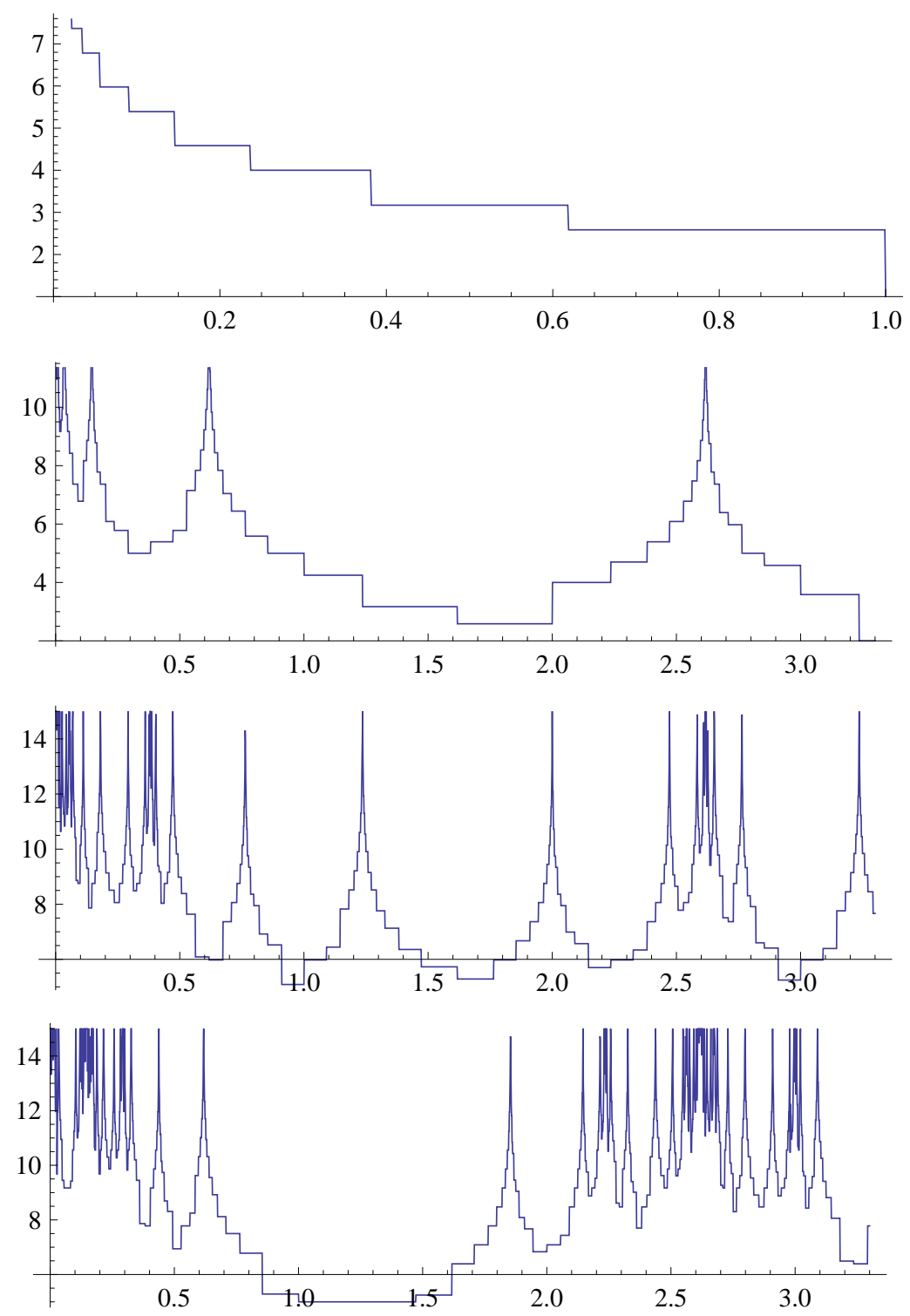

Figure 2. Self-similar cascades defined over $\mathbb{Q}(\sqrt{5})$. 
This means $B$ is locally invariant under a linear contraction fixing $a$, for every $a \in B$.

A similar renormalization mechanism causes one bifurcation set to reappear in another. Examples of four such bifurcation sets, with the periods of $\rho_{0}$ in $\mathbb{Q}(\sqrt{5})$, are shown in Figure 2. Note that a compressed version of each cascade reappears in the next, near $t=(3+\sqrt{5}) / 2=2.618 \ldots$ Using this idea we give explicit examples where $\omega^{k}$ embeds in $B(\S 10)$.

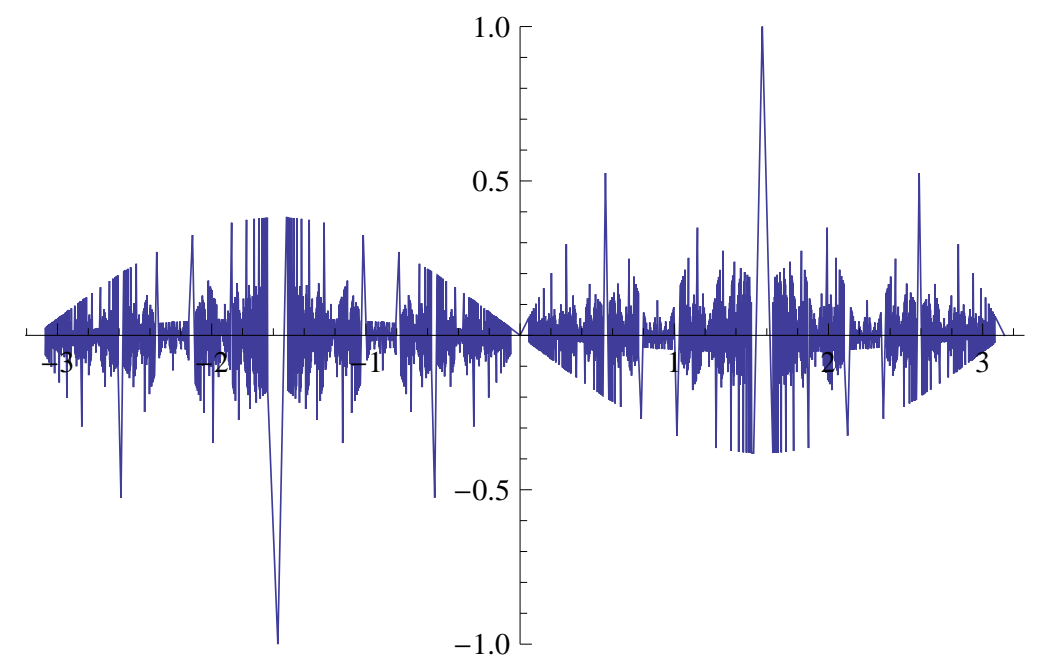

Figure 3. Fluctuations of $C\left(\rho_{t}\right)$ for billiards in a regular pentagon.

Appendix: Teichmüller curves. Much attention has focused on the family of foliations arising from parallel geodesics with varying slopes on the flat surface $(X,|\omega|)$. These foliations are given by $\rho_{t}=\operatorname{Re}\left(e^{i t} \omega\right)$.

The invariants discussed in $\S 2$ can also be studied for such families. In the Appendix we show that the (rapidly fluctuating) convex set $C\left(\rho_{t}\right) \subset$ $H^{1}(X)$ can be readily determined whenever $(X, \omega)$ generates a Teichmüller curve. An example associated to billiards in the regular pentagon is shown in Figure 3. This convex set records the cylinder decomposition at each cusp of $\mathrm{SL}(X, \omega)$, and can be computed by a continued fraction algorithm in $\mathbb{Q}(\sqrt{5})$. For another perspective, we also show:

The function graphed in Figure 3 arises from the radial limits $F: S^{1} \rightarrow \mathbb{P} \mathcal{M L}_{g}$ of a suitable Teichmüller disk $f: \Delta \rightarrow \mathcal{T}_{g}$.

Thus we have an explicit example of the discontinuous boundary behavior of Teichmüller disks in Thurston's compactification of $\mathcal{T}_{g}$. 
Notes and references. For $n=2$, the invariant $\operatorname{flux}(S, \rho) \in H_{n-1}(E)$ packages the same information as the SAF invariant of an associated interval exchange transformation, and is related to the Kenyon-Smillie $J$-invariant. For these and other perspectives on foliations, flux and the period torus, see e.g. [Ar1], [Ar2], [Nov], [Fr], [V1], [Fa], [KS, §4], [Z] and [Mc2, §4]. Theorem 1.1 strengthens [Ar1, Thm 3.7]. For a related result, see [Mc2, Thm. 2.1].

The families $\left(X_{t}, \rho_{t}\right)$ in Theorem 1.2 are transverse to the orbits of $\mathrm{SL}_{2}(\mathbb{R})$ acting on $\Omega \mathcal{M}_{g}$. They correspond instead to the leaves of the absolute period foliation. The self-similarity of parameter space for quadratic periods, formulated as Theorem 1.4 above, is a reflection of the quasiconformal action of $\mathrm{SL}_{2} \mathcal{O}_{D}$ associated to Hilbert modular surfaces; see [Mc6, $\S 8]$ and [Mc8]. The present approach aims to place this phenomena into a broader dynamical setting. Applications to isoperiodic forms are developed in [Mc9], and served as the original motivation for this paper.

I am grateful to P. Arnoux and the referees for many helpful remarks and references, and to E. Lanneau for the second example in $\S 5$.

\section{Invariants of foliations}

Let $(X, \rho)$ be a closed, oriented $n$-manifold equipped with a smooth, closed 1 -form $\rho \neq 0$. The form $\rho$ determines a codimension-one measured foliation $\mathcal{F}(\rho)$ of $X$ (with singularities on its zero set $Z(\rho)$ ).

In this section we describe several invariants of the pair $(X, \rho)$. Throughout, $H^{*}(X)$ denotes cohomology with real coefficients.

The period torus. The periods of $(X, \rho)$ are given by

$$
P=\operatorname{Per}(X, \rho)=\left\{\int_{C} \rho: C \in H_{1}(X, \mathbb{Z})\right\} \subset \mathbb{R} .
$$

We have $P \cong \mathbb{Z}^{r}$ for some $r \geq 0$, which we call the rank of $(X, \rho)$. Choosing a basis, we can write $P=\oplus_{1}^{r} \mathbb{Z} a_{i}$ where $a_{1}, \ldots, a_{r} \in \mathbb{R}$ are linearly independent over $\mathbb{Q}$. There is a corresponding expression for $[\rho] \in H^{1}(X)$ as a linear combination of integral cohomology classes

$$
[\rho]=\sum_{1}^{r} a_{i}\left[\xi_{i}\right], \quad\left[\xi_{i}\right] \in H^{1}(X, \mathbb{Z}),
$$

represented by smooth forms $\left(\xi_{i}\right)$. The period map

$$
\pi: X \rightarrow E \cong \mathbb{R}^{r} / \mathbb{Z}^{r}
$$


is defined by

$$
\pi(x)=\left(\int_{p}^{x} \xi_{1}, \ldots, \int_{p}^{x} \xi_{r}\right)
$$

where $p$ is a basepoint in $X$. Note that $\xi_{i}=\pi^{*}\left(d x_{i}\right)$, where $\left(x_{1}, \ldots, x_{r}\right)$ are the standard coordinates on $\mathbb{R}^{r}$.

The target of $\pi$ is the period torus, defined intrinsically by:

$$
E=\left(P \otimes_{\mathbb{Z}} \mathbb{R}\right) / P \cong H^{1}(X, \mathbb{R})_{\rho}^{*} / \operatorname{Im}\left(H_{1}(X, \mathbb{Z})\right),
$$

where $H^{1}(X, \mathbb{R})_{\rho}$ is the smallest subspace of $H^{1}(X)$ defined over $\mathbb{Q}$ and containing $[\rho]$.

Foliations and flux. Via Poincaré duality, $[\rho]$ determines a class

$$
[\mathcal{F}(\rho)] \in H_{n-1}(X)
$$

characterized by

$$
\langle\mathcal{F}(\rho),[\alpha]\rangle=\int_{X} \rho \wedge \alpha
$$

for any $[\alpha] \in H^{n-1}(X)$. Geometrically, this class records the diffuse oriented $(n-1)$-manifold coming from the leaves of $\mathcal{F}(\rho)$ (cf. [Sul]).

The flux of the foliation is defined by

$$
\operatorname{flux}(X, \rho)=\pi_{*}([\mathcal{F}(\rho)]) \in H_{n-1}(E) .
$$

It records the average distribution of the leaves of $\mathcal{F}(\rho)$ under the period map.

Note that we may choose the forms $\xi$ to satisfy $\rho=\sum_{1}^{r} a_{i} \xi_{i}$. Then the period map sends the leaves of $\mathcal{F}(\rho)$ into the leaves of the foliation $\mathcal{F}\left(\rho^{\prime}\right)$ of $E \cong \mathbb{R}^{r} / \mathbb{Z}^{r}$ defined by $\rho^{\prime}=\sum a_{i} d x_{i}$. Since the real numbers $\left(a_{1}, \ldots, a_{r}\right)$ are linearly independent over $\mathbb{Q}, \mathcal{F}\left(\rho^{\prime}\right)$ gives an irrational foliation of $E$; that is, its leaves are parallel immersed copies of $\mathbb{R}^{r-1}$. (When $r=1, E=S^{1}$ and each leaf is a single point.)

The alternating form. The flux carries the same information as the integral alternating form

$$
\pi^{*}: \wedge^{n} H^{1}(E, \mathbb{Z}) \cong H^{n}(E, \mathbb{Z}) \rightarrow H^{n}(X, \mathbb{Z}) \cong \mathbb{Z} .
$$

Indeed, from the flux we can obtain the real numbers

$$
\left\langle\operatorname{flux}(X, \rho), d x_{i_{2}} \ldots d x_{i_{n}}\right\rangle=\sum_{1}^{r} a_{i} \int_{X} \xi_{i} \xi_{i_{2}} \ldots \xi_{i_{n}},
$$


which in turn determine the alternating form since the coefficients $\left(a_{i}\right)$ are linearly independent over $\mathbb{Q}$.

Degree. Now suppose the rank of $(X, \rho)$ and the dimension of $X$ agree. Choose an orientation for $E$; for concreteness, say it is defined by the unit volume form $d x_{1} \ldots d x_{n}$. We then define the degree of the foliation by

$$
\operatorname{deg}(X, \rho)=\operatorname{deg}(\pi: X \rightarrow E)=\int_{X} \xi_{1} \ldots \xi_{n} \in \mathbb{Z} .
$$

This integer is also implicit in the flux; it satisfies

$$
\operatorname{deg}(X, \rho)=\left(1 / a_{1}\right)\left\langle\operatorname{flux}(X, \rho),\left[d x_{2} \ldots d x_{n}\right]\right\rangle .
$$

Dynamical invariants. The flux and the homotopy class of the period map depend only on the cohomology class $[\rho] \in H^{1}(X)$. We now turn to invariants which can distinguish different foliations in the same cohomology class.

Let $f \in L^{\infty}(X)$ be a measurable function with $0 \leq f \leq 1$, such that $d f \wedge \rho=0$ as a current. This simply means that $f$ is constant a.e. along the leaves of $\mathcal{F}(\rho)$. Then $d(f \rho)=0$, so $[f \rho]$ defines a class in $H^{1}(X)$ (cf. [GH, Ch. 3.1]). We define the content of $\rho$ by

$$
C(\rho)=\{[f \rho]: d f \wedge \rho=0 \text { and } 0 \leq f \leq 1\} \subset H^{1}(X) .
$$

Since the unit ball in $L^{\infty}(X)$ is compact as a space of distributions, we find:

$$
C(\rho) \text { is a compact, convex subset of } H^{1}(X) \text {. }
$$

Note that $C(\rho)$ satisfies the linear constraint $[\rho] \wedge C(\rho)=0 \in H^{2}(X)$.

Saturation and ergodicity. A measurable set $A \subset X$ is saturated if it is a union of leaves of $\mathcal{F}(\rho)$; equivalently, if $d\left(\chi_{A} \rho\right)=0$, where $\chi_{A}$ is the indicator function of $A \subset X$.

The foliation defined by $(X, \rho)$ is ergodic if every saturated set has zero or full measure. In this case, $C(\rho)=[0,1] \cdot[\rho]$. In general, $C(\rho)$ is the closed convex hull of the classes $\left[\chi_{A} \rho\right] \in H^{1}(X)$ coming from saturated sets $A \subset X$.

Note that $C(\rho)$ is only sensitive to the Lebesgue measure class; it does not record currents represented by singular transverse invariant measures for $\mathcal{F}(\rho)$.

Relative degree and flux. The relative flux of a saturated set $A \subset X$ is the cycle in $H_{n-1}(E)$ characterized by

$$
\langle\operatorname{flux}(A, \rho), \alpha\rangle=\int_{A} \rho \wedge \pi^{*}(\alpha)
$$


for all $[\alpha] \in H^{n-1}(E)$. Equivalently, the relative flux is the image of $\left[\chi_{A} \rho\right]$ under the map $H^{1}(X) \cong H_{n-1}(X) \rightarrow H_{n-1}(E)$.

In the case $r=n$, we define the relative degree of a saturated set by

$$
\operatorname{deg}(A, \rho)=\int_{A} \xi_{1} \ldots \xi_{n} .
$$

The relative degree and flux are related by (2.5), with $X$ replaced by $A$. For any countable partition of $X$ into saturated sets, we have

$$
\operatorname{deg}(X, \rho)=\sum \operatorname{deg}\left(A_{i}, \rho\right)
$$

The real numbers on the right record how the topological degree of $X / E$ is apportioned among the measurable sets $A_{i}$.

Upper and lower degrees. The extreme values of the relative degree will be denoted by

$$
\operatorname{deg}^{-}(X, \rho)=\inf _{A} \operatorname{deg}(A, \rho) \text { and } \operatorname{deg}^{+}(X, \rho)=\sup _{A} \operatorname{deg}(A, \rho) .
$$

Since $(X-A)$ is saturated whenever $A$ is saturated, we have

$$
\operatorname{deg}^{-}(X, \rho)+\operatorname{deg}^{+}(X, \rho)=\operatorname{deg}(X, \rho) .
$$

These extreme values can also be expressed in terms of $C(\rho)$; for example, we have

$$
\operatorname{deg}^{+}(X, \rho)=\sup \left\{\frac{1}{a_{1}} \int_{X} \alpha \xi_{2} \ldots \xi_{n}:[\alpha] \in C(\rho)\right\} .
$$

Properties of invariants. The rank and the degree of $(X, \rho)$, and more generally the homotopy class of $\pi: X \rightarrow E$, only depend on the cohomology class $[\rho] \in H^{1}(X)$. These invariants are also homogeneous of degree zero they are unchanged if $\rho$ is multiplied by a scalar.

The invariants $C(\rho)$ and $\operatorname{deg}^{ \pm}(X, \rho)$, on the other hand, depend on the form $\rho$ and the dynamics of its measured foliation. They are homogeneous of degree one and zero respectively; that is,

$$
C(\lambda \rho)=\lambda \cdot C(\rho) \text { and } \operatorname{deg}^{ \pm}(X, \lambda \rho)=\operatorname{deg}^{ \pm}(X, \rho) .
$$

for $\lambda>0$.

Proposition 2.1 If $\rho_{n} \rightarrow \rho$ in the $C^{\infty}$ topology, then we have

$$
\lim \sup C\left(\rho_{n}\right) \subset C(\rho)
$$

as compact subsets of $H^{1}(X)$. 
This means the limit of every convergent subsequence $x_{n_{k}} \in C\left(\rho_{n_{k}}\right)$ lies in $C(\rho)$.

Proof. Suppose $\left[f_{n} \rho_{n}\right] \in C\left(\rho_{n}\right)$ converges, along a subsequence, to $[\alpha] \in$ $H^{1}(X)$. Passing to a further subsequence we can assume $f_{n} \rightarrow f$ weakly, where $0 \leq f \leq 1$. Then $d(f \rho)=\lim d\left(f_{n} \rho_{n}\right)=0$, so $\left[f_{n} \rho_{n}\right] \rightarrow[f \rho] \in C(\rho)$.

Using equation (2.7) we obtain:

Corollary 2.2 If $\rho_{n} \rightarrow \rho$ and $\left[\rho_{n}\right]=[\rho]$ for all $n$, then

$$
\limsup \operatorname{deg}^{+}\left(X, \rho_{n}\right) \leq \operatorname{deg}^{+}(X, \rho) .
$$

A similar statement holds for the lower degree.

\section{Harmonic foliations on Riemann surfaces}

In this section we discuss the invariants of $\S 2$ in the case of a harmonic form on a compact Riemann surface.

Holomorphic and harmonic forms. Let $X$ be a compact Riemann surface of genus $g$, and let $\mathcal{H}(X)$ and $\Omega(X)$ denote the spaces of harmonic and holomorphic 1-forms on $X$. We have natural isomorphisms

$$
\Omega(X) \cong \mathcal{H}(X) \cong H^{1}(X),
$$

given by $\omega \mapsto \rho=\operatorname{Re}(\omega) \mapsto[\rho]$. The period map $\pi: X \rightarrow E$ can be factored through the Abel-Jacobi map

$$
X \rightarrow \operatorname{Jac}(X) \cong \mathcal{H}(X)^{*} / H_{1}(X, \mathbb{Z}),
$$

provided we use harmonic forms for $\left(\xi_{1}, \ldots, \xi_{r}\right)$.

Moduli spaces. The moduli space $\Omega \mathcal{M}_{g}$ of nonzero holomorphic forms $(X, \omega)$ of genus $g$ has been much studied (cf. [KZ], [Mo2], [Mc8]). It decomposes into strata $\Omega \mathcal{M}_{g}\left(p_{i}\right)$ where the multiplicities of the zeros of $\omega$ give a fixed partition $\left(p_{i}\right)$ of $2 g-2$. Each stratum has local coordinates given by the relative period map

$$
\pi:(X, \omega) \mapsto[\omega] \in H^{1}(X, Z(\omega) ; \mathbb{C}) .
$$

The moduli space $\mathcal{H} \mathcal{M}_{g}$ of harmonic forms $(X, \rho)$ is isomorphic to $\Omega \mathcal{M}_{g}$ via the relation $\rho=\operatorname{Re}(\omega)$. 
Cylinders and saddle connections. Let $(X, \rho)$ be a harmonic form of genus $g$. Its zero set satisfies $|Z(\rho)| \leq 2 g-2$. A leaf of $\mathcal{F}(\rho)$ which joins a pair of zeros (or a single zero to itself) is called a saddle connection. A cylinder is a maximal open region $U \subset X$ such that $\mathcal{F}(\rho) \mid U$ is homeomorphic to $S^{1} \times(0,1)$ with the product foliation by circles.

Cylindrical and minimal components. The topological dynamics of $\mathcal{F}(\rho)$ gives a natural decomposition of $X$ into components $\left(X_{i}\right)_{i=1}^{s}$, characterized by the following properties.

1. The components $\left(X_{i}\right)$ have disjoint, nonempty interiors, and $X=$ $\bigcup_{1}^{s} X_{i}$.

2. Each component $X_{i}$ is either the closure of a cylinder, or the closure of a single (infinite) leaf of $\mathcal{F}(\rho)$. In the first case $X_{i}$ is cylindrical; in the second case it is minimal.

See e.g. [Str, §11.4], [Z, §2.1].

We say $(X, \rho)$ is periodic if all its components are cylindrical, and minimal if $\mathcal{F}(\rho)$ has a dense leaf (in which case $X=X_{1}$ ).

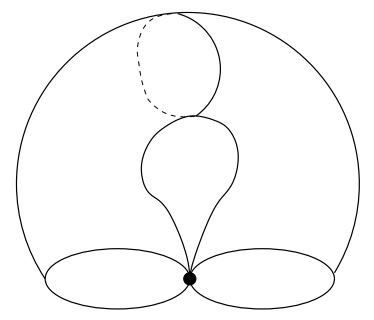

Figure 4. A cylinder with two boundary points identified at a zero of $\rho$.

Topology of components. The boundary of each component $X_{i}$ is a finite union of saddle connections. Thus $X_{i}$ itself is a compact, connected subsurface of $X$, apart from boundary identifications at the zeros of $\rho$ (see Figure 4). The double of the interior of $X_{i}$ has genus $g_{i} \geq 1$, since it carries a nonzero harmonic form. Thus its Euler characteristic satisfies $\chi\left(\operatorname{int} X_{i}\right)=$ $1-g_{i} \leq 0 ;$ moreover

$$
\chi(X) \leq \sum \chi\left(\operatorname{int} X_{i}\right)
$$

since $\chi\left(\bigcup \partial X_{i}\right) \leq 0$. Finally we note that

$$
X_{i} \text { minimal } \Longrightarrow \operatorname{int} X_{i} \text { has genus } \geq 1 \text {. }
$$


Otherwise $\rho \mid \operatorname{int} X_{i}$ would be exact, and hence the leaves of $\mathcal{F}(\rho) \mid \operatorname{int} X_{i}$ would be compact.

Degree and flux. With respect to a basis $\left(\xi_{i}\right)_{i=1}^{r}$ of $H^{1}(X, \mathbb{Z})_{\rho}$, we have

$$
\operatorname{flux}(X, \rho)=\left(f_{1}, \ldots, f_{r}\right)=\left(\int_{X} \rho \wedge \xi_{i}\right)_{i=1}^{r} \in H_{1}(E) .
$$

The coordinate $f_{i}$ measures the average drift of the leaves of $\mathcal{F}(\rho)$ in the $x_{i}$ direction, when projected to $E=\mathbb{R}^{r} / \mathbb{Z}^{r}$. Since the components $\left(X_{i}\right)$ are essentially disjoint, we have

$$
\operatorname{flux}(X, \rho)=\sum_{i=1}^{s} \operatorname{flux}\left(X_{i}, \rho\right) .
$$

Proposition 3.1 The flux vanishes on the cylindrical components of $X$.

Proof. Let $L_{i} \cong S^{1}$ be a smooth closed leaf of $\mathcal{F}(\sigma)$ lying in the interior of a cylindrical component $X_{i} \subset X$. Since $X_{i}$ is swept out by parallel copies of $L_{i}$, the cycle $\left[\rho \mid X_{i}\right] \in H_{1}(X)$ is a multiple of $\left[L_{i}\right]$, and hence flux $\left(X_{i}, \rho\right)$ is a multiple of $\left[\pi\left(L_{i}\right)\right] \in H_{1}(E)$. As remarked in $\S 2$, we may choose $\pi$ so it sends the leaves of $\mathcal{F}(\rho)$ into the leaves of an irrational foliation of $E$. Thus $\pi\left(L_{i}\right)$ is contained in an immersed copy of $\mathbb{R}^{r-1}$, so $\left[\pi\left(L_{i}\right)\right]=0$.

Corollary 3.2 If $(X, \rho)$ is periodic, then $\operatorname{flux}(X, \rho)=0$ and $\operatorname{rank}(X, \rho) \leq g$.

Proof. We have flux $(X, \rho)=\sum_{1}^{s} \operatorname{flux}\left(X_{i}, \rho\right)=0$; hence by (2.4), the restriction of the symplectic form to $H^{1}(X)_{\rho}$ is zero.

Proposition 3.3 If $(X, \rho)$ has rank two, then $\operatorname{deg}\left(X_{i}, \rho\right) \in \mathbb{Z}$ for all $i$.

Proof. Since $[\rho]=0$ in $H^{1}\left(\partial X_{i}\right)$, the same is true for $\left[\xi_{i}\right]$. Thus we can choose the forms $\left[\xi_{i}\right]$ such that $\rho=a_{1} \xi_{1}+a_{2} \xi_{2}$ and $\xi_{i} \mid \partial X_{i}=0$. With this normalization, $\pi \mid \partial X_{i}$ is locally constant. Thus $\pi\left(\partial X_{i}\right)=E_{0}$ is a finite subset of $E$, and the map

$$
\pi^{*}: H^{2}(E) \cong H^{2}\left(E, E_{0}\right) \rightarrow H^{2}\left(X_{i}, \partial X_{i}\right)
$$


is given by a multiplication by an integer. This integer can be computed as the pullback of the fundamental class $[E]$, which is the same as the relative degree

$$
\operatorname{deg}\left(X_{i}, \rho\right)=\int_{X_{i}} \xi_{1} \wedge \xi_{2}
$$

Remark: Laminations and closed 1-forms. Similar discussions can be made for more general closed 1-forms on surfaces, and for suitable geodesic laminations.

The leaves of $\mathcal{F}(\rho)$ can be canonically straightened to yield an oriented, measured geodesic lamination $\lambda(\rho)$ on $X$; see e.g. [Le2]. The invariants $C(\rho)$ and $\pi: X \rightarrow E$ could also be defined directly in terms of this lamination, and the minimal and cylindrical pieces of $\mathcal{F}(\rho)$ correspond simply to the components of $\lambda(\rho)$.

Note, however, that not every oriented measured geodesic lamination $\lambda$ arises from a harmonic 1-form; for example, its finite sublaminations must be nontrivial in $H^{1}(X, \mathbb{Z})$. This tautness condition is also sufficient, by Calabi's characterization of harmonic 1-forms [Ca]. Calabi's result also shows that the study of a general 1-form $\rho$ on a smooth surface, assumed to have standard, isolated zeros, can be reduced to the harmonic case by performing Whitehead moves and collapsing cylinders. See $[Z, \S 2.2]$ for more details.

\section{Recurrence, divergence and flux}

Let $\pi: X \rightarrow E$ be the period map for a harmonic 1-form $\rho$ on a compact Riemann surface $X$. The universal cover of $E$ determines the smallest covering space $\widetilde{X} \rightarrow X$ on which $\rho$ becomes exact. Thus we have a commutative diagram:

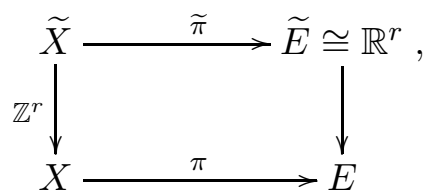

where $\tilde{\pi}$ is a proper map. Let $\widetilde{\rho}$ denote the pullback of $\rho$ to $\widetilde{X}$. We begin this section by observing:

Theorem 4.1 Every recurrent leaf of $\mathcal{F}(\widetilde{\rho})$ is actually periodic. 
We then discuss the rate at which aperiodic leaves diverge to infinity, and relate the flux of $(X, \rho)$ to the average behavior of leaves.

Leaves and hyperplanes. As in $\S 2$, it is convenient to choose $\pi$ so that $\widetilde{\rho}=\widetilde{\pi}^{*}\left(\sum a_{i} d x_{i}\right)$, where $\left(x_{1}, \ldots, x_{r}\right)$ are standard coordinates on $\widetilde{E} \cong \mathbb{R}^{r}$. Then $\widetilde{\pi}$ sends the leaves of $\mathcal{F}(\widetilde{\rho})$ into level sets of the linear function $\sum a_{i} x_{i}$ on $\mathbb{R}^{r}$.

It is also useful to introduce the conformal metric $|\omega|$ on $X$, where $\omega \in$ $\Omega(X)$ is the unique holomorphic 1-form satisfying $\rho=\operatorname{Re}(\omega)$. In this metric, the unit speed flow along the leaves of $\mathcal{F}(\rho)$ preserves the measure $|\omega|^{2}$.

The lifted metric $|\widetilde{\omega}|$ on $\widetilde{X}$ provides, for each $p \in \widetilde{X}$, a natural map

$$
L: \mathbb{R} \rightarrow \tilde{X}
$$

sweeping out the leaf of $\mathcal{F}(\widetilde{\rho})$ through $p$ at unit speed. In local coordinates on $\widetilde{X}$ where $\widetilde{\omega}=d z$, the leaves of $\mathcal{F}(\widetilde{\rho})$ are vertical lines and $L^{\prime}(t)=i$.

We say the leaf through $p$ is recurrent iff $L(t)$ returns infinitely often (as $|t| \rightarrow \infty)$ to a fixed compact set $K \subset \widetilde{X}$.

Proof of Theorem 4.1. Let us say a domain $R \subset \widetilde{X}$ is a rectangle if $(R, \omega)$ is isomorphic to $([0, a] \times[0, b], d z)$ for some $a, b>0$. Cover $X$ by a finite number of rectangles, and then lift them to obtain a locally finite covering of $\widetilde{X}$ by countably many rectangles $\left(R_{i}\right)$.

Suppose $L(t)$ is recurrent. Then it returns infinitely often to some rectangle $R_{i} \cong[0, a] \times[0, b]$. Thus we can find $t_{1}<t_{2}$ such that $L\left(t_{i}\right)=\left(a_{i}, b / 2\right)$ in this chart for $i=1,2$. Adjoining to $L\left[t_{1}, t_{2}\right]$ the horizontal segment $\left[a_{1}, a_{2}\right] \times\{b / 2\}$, we obtain a 1 -cycle $C \subset \widetilde{X}$ satisfying

$$
b_{2}-b_{1}=\int_{C} \tilde{\rho}
$$

Since $\widetilde{\rho}$ is exact on $\widetilde{X}$, we have $b_{1}=b_{2}$ and hence $L(t)$ is periodic.

Let $\|\cdot\|$ denote the Euclidean norm on $\widetilde{E} \cong \mathbb{R}^{r}$. Since $\widetilde{\pi}$ is proper, we have:

Corollary 4.2 For any aperiodic leaf of $\mathcal{F}(\widetilde{\rho})$, we have $\|\widetilde{\pi}(L(t))\| \rightarrow \infty$ as $t \rightarrow \infty$.

To strengthen this result, we show that an aperiodic leaf spends a bounded amount of time in any unit ball. 
Theorem 4.3 There exists a $T>0$ such that

$$
|\{t: \tilde{\pi}(L(t)) \in B(x, 1)\}| \leq T
$$

for all $x \in \mathbb{R}^{r}$ and all aperiodic leaves of $\mathcal{F}(\widetilde{\rho})$.

Proof. By properness and $\mathbb{Z}^{r}$-periodicity of $\tilde{\pi}$, there exists an $N>0$ such that for all $x \in \mathbb{R}^{r}$, the preimage of $B(x, 1)$ in $\widetilde{X}$ can be covered by $\leq N$ rectangles drawn from the covering $\left(R_{i}\right)$ constructed in the proof of Theorem 4.1. An aperiodic leaf can visit each of these rectangles at most once. Thus the amount of time a leaf can spend in such a ball is bounded by $T=N h$, where $h$ is the maximum height of a rectangle in the covering $\left(R_{i}\right)$.

Corollary 4.4 There exists a $C>0$ such that

$$
\limsup _{t \rightarrow \infty} \frac{\|\tilde{\pi}(L(t))\|^{r-1}}{t}>C
$$

whenever $L(t)$ is aperiodic.

Proof. Any leaf of $\mathcal{F}(\widetilde{\rho})$ such as $L(\mathbb{R})$ projects under $\widetilde{\pi}$ into a hyperplane of the form $\sum a_{i} x_{i}=b$. Thus its intersection with the ball of radius $M$ in $\mathbb{R}^{r}$ can be covered by $O\left(M^{r-1}\right)$ unit balls. The projected leaf spends only a bounded amount of time in each such ball, so we have $\mid \widetilde{\pi}(L(t) \mid>M$ for some $t>0$ with $t=O\left(M^{r-1}\right)$.

Flux. We now show that the average drift of the leaves of $\mathcal{F}(\widetilde{\rho})$ is described by the flux of $(X, \rho)$.

Let $\alpha$ be a smooth 1 -form on $X$, and let $\mathcal{L}(x, T)$ denote the segment of length $T$ along the leaf of $\mathcal{F}(\rho)$ beginning at $x \in X$. The unit speed flow along the leaves of $\mathcal{F}(\rho)$ preserves the measure $|\omega|^{2}$. Thus by the ergodic theorem, the limit

$$
F(x)=\lim _{T \rightarrow \infty} \frac{1}{T} \int_{\mathcal{L}(x, T)} \alpha
$$

exists for a.e. $x \in X$, and satisfies

$$
\int_{X} \rho \wedge \alpha=\int_{X} F(x)|\omega|^{2}
$$

The same equation holds with $X$ replaced by any saturated set $A \subset X$ of positive measure.

Applying this observation to the forms $\xi_{i}=d x_{i}, i=1, \ldots, r$, we obtain: 
Theorem 4.5 For almost every $x \in X$, the limit

$$
f(x)=\lim _{t \rightarrow \infty} \frac{\tilde{\pi}(L(t))}{t} \in H_{1}(E) \cong \mathbb{R}^{r}
$$

exists for any leaf of $\mathcal{F}(\widetilde{\rho})$ lying above $x$, and all such leaves give the same limit. The function $f: X \rightarrow H_{1}(E)$ is constant along the leaves of $\mathcal{F}(\rho)$, and satisfies

$$
\operatorname{flux}(A, \rho)=\int_{A} f(x)|\omega|^{2}
$$

for any saturated set $A \subset X$.

Asymptotic cycles. We can regard $f(x) \in H_{1}(E)$ as the homology class of the asymptotic 1-cycle determined by the leaf of $\mathcal{F}(\rho)$ through $x$. If $\mathcal{F}(\rho)$ is uniquely ergodic, $f(x)$ is constant.

By Theorem 4.5, a foliation $\mathcal{F}(\rho)$ has zero flux iff the leaves of $\mathcal{F}(\widetilde{\rho})$ have no directional drift on average. Individual leaves can still have definite drift, so long as these effects cancel. For related discussions, see [Sch] and [Fr].

\section{Cubic examples}

This section describes two examples of minimal foliations with zero flux. In these examples the leaves of $\mathcal{F}(\widetilde{\rho})$ meander slowly to infinity without closing, as shown in Figure 5. More precisely, we have

$$
|\widetilde{\pi}(L(t))| \rightarrow \infty \text { but } \tilde{\pi}(L(t)) / t \rightarrow 0 .
$$

Both examples are associated to cubic number fields and pseudo-Anosov mappings on surfaces of genus three. Such examples cannot exist in genus two, as we will see in the sections that follow.

Eigenvectors and flux. Let $f: X \rightarrow X$ be an orientation-preserving homeomorphism on a closed surface of genus $g$, and let

$$
P(t)=\operatorname{det}(t I-F),
$$

where $F: H^{1}(X, \mathbb{Z}) \rightarrow H^{1}(X, \mathbb{Z})$ is the induced map on cohomology. Here are two natural sources of foliations with zero flux.

(i) If $P(t)$ is irreducible, then $\operatorname{flux}(X, \rho)=0$ for any eigenvector $[\rho]$ of $T=F+F^{-1}$. To see this, observe that the minimal polynomial for $T$ is irreducible and of degree $g$. Moreover the symplectic form satisfies 

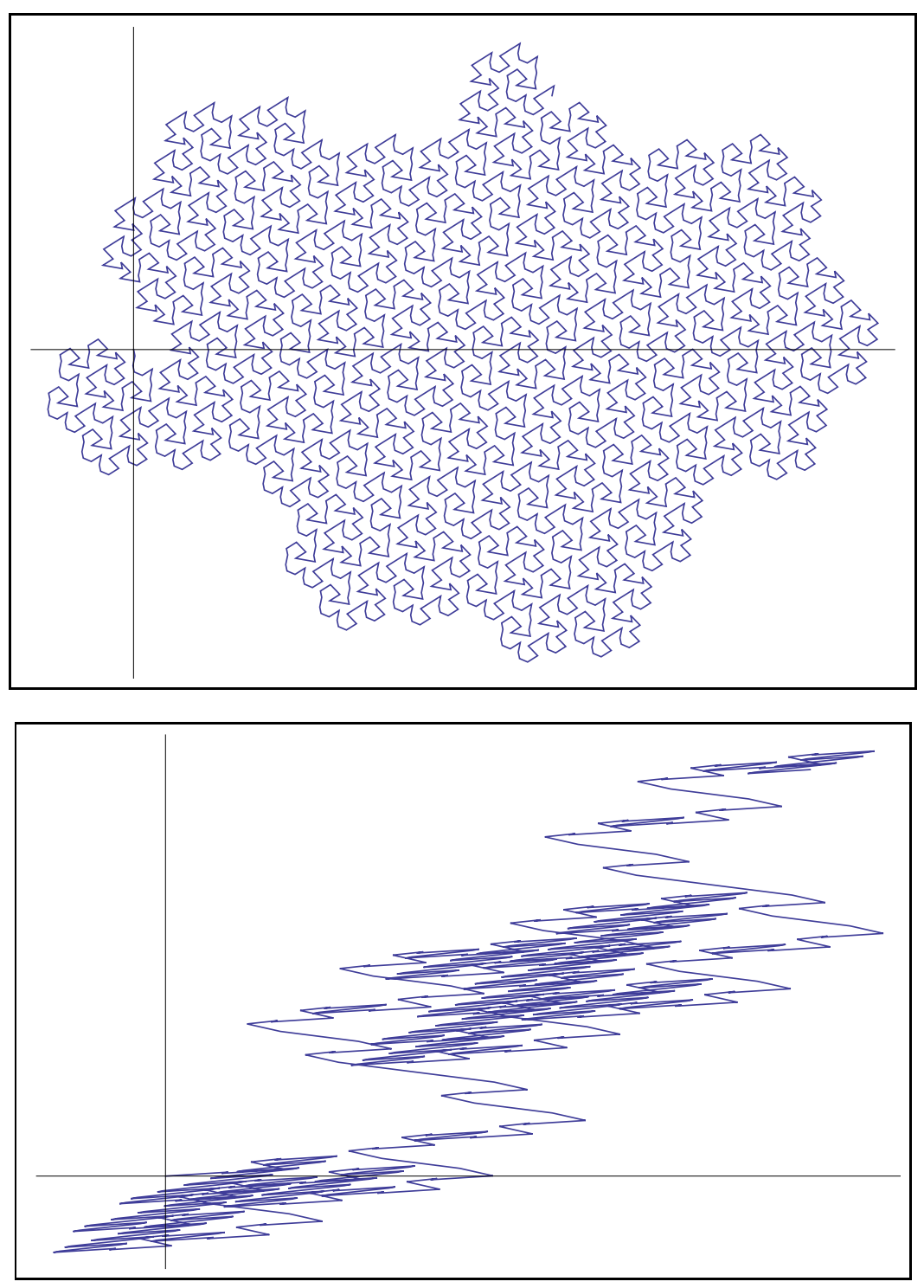

Figure 5. Zero flux leaves for cubic fields. 
$\langle T x, x\rangle=0$ for all $x \in H^{1}(X)$. Since $H^{1}(X)_{\rho}$ is spanned by an orbit of $T$, it is Lagrangian, and hence the flux of $\rho$ is zero.

The same reasoning shows that $\operatorname{flux}(X, \rho)=0$ whenever $K$ is a totally real field of degree $g$, and $[\rho] \in H^{1}(X, K)$ is the real part of a holomorphic eigenform for real multiplication by $K$. For more on real multiplication, see e.g. [Mc1], [Mc2] and [Mo1].

(ii) Suppose, on the other hand, that

$$
P(t)= \pm Q(t) t^{g} Q(1 / t)
$$

is a product of distinct irreducible polynomials of degree $g$, where the roots of one are the reciprocals of the roots of the other. (The sign is chosen so that both $P$ and $Q$ are monic polynomials.) Then $\operatorname{Ker} Q(F)$ and $\operatorname{Ker} Q\left(F^{-1}\right)$ are complementary Lagrangian subspaces of $H^{1}(X)$, so any real eigenvector of $F$ has zero flux.

I. Complex cubic example. An example where $f$ is a pseudo-Anosov mapping on a surface of genus 3, satisfying (5.1) with $Q(t)=t^{3}-t^{2}-t-1$, was given by Arnoux and Yoccoz $[\mathrm{AY}]$.

Let $K=\mathbb{Q}(\lambda \subset \mathbb{R})$ be the cubic field generated by the unique real root $\lambda>1$ of $Q(t)$. Let $\lambda^{\prime}$ be one of the two remaining complex roots. Then we have a natural embedding $K \cong \mathbb{Q}\left(\lambda^{\prime}\right) \rightarrow \mathbb{C}$, which we will denote by $x \mapsto x^{\prime}$. Note that $\left|\lambda^{\prime}\right|=\lambda^{-1 / 2}$.

The pseudo-Anosov representative of the mapping-class $[f]$ gives a holomorphic 1-form $(X, \omega)$ of genus three, and a map $f: X \rightarrow X$ such that

$$
f(x+i y)=\lambda x+i \lambda^{-1} y
$$

in local coordinates where $\omega=d z, z=x+i y$. Thus $\rho=\operatorname{Re}(\omega)$ satisfies $f^{*}(\rho)=\lambda \rho$. By general properties of pseudo-Anosov mappings, $\mathcal{F}(\rho)$ is minimal and even uniquely ergodic [FLP].

Let $\widetilde{f}$ be a lift of $f$ to the $\mathbb{Z}^{3}$-periodic cover $\widetilde{X} \rightarrow X$ studied in $\S 4$. Since $Q(t)$ has no roots on $S^{1}$, we can choose the period map (within its homotopy class) so that

$$
\widetilde{\pi}: \widetilde{X} \rightarrow \widetilde{E} \cong H^{1}(X)_{\rho}^{*}
$$

conjugates $\tilde{f}$ to the linear action of $f_{*}$ on homology ([Fra]; see also [Ar2], $[\mathrm{Fa}])$. The action of $f_{*}$ makes $H^{1}(X)_{\rho}$ into a 1-dimensional vector space over $K$. Choosing a basis, we obtain an isomorphism

$$
\widetilde{E} \cong K \otimes_{\mathbb{Q}} \mathbb{R} \cong \mathbb{R} \oplus \mathbb{C}
$$


We can regard the complex factor as the $\lambda^{\prime}$ eigenspace for $f_{*}$. Composing $\tilde{\pi}$ with projection onto this factor, we obtain a map

$$
\phi: \widetilde{X} \rightarrow \mathbb{C}
$$

satisfying

$$
\phi(\widetilde{f}(x))=\lambda^{\prime} \phi(x) .
$$

The form $\widetilde{\rho}$ is the differential of projection of $\widetilde{E} \cong \mathbb{R} \oplus \mathbb{C}$ to its real factor, so this projection sends leaves to points. Thus we have

$$
\operatorname{diam} \tilde{\pi}(L) \asymp \operatorname{diam} \phi(L)
$$

whenever $L$ is contained in a leaf of $\mathcal{F}\left(\rho_{t}\right)$.

Proposition 5.1 All sufficiently long segments $L$ of the leaves of $\mathcal{F}(\widetilde{\rho})$ satisfy

$$
\operatorname{diam}(\widetilde{\pi}(L)) \asymp \operatorname{length}(L)^{1 / 2} .
$$

Here the length is defined by $\int_{L}|\widetilde{\omega}|$.

Proof. Choose a compact set $M \subset \widetilde{X}$ such that every segment of unit length in $X$ has a lift to $M$. Let $L$ be a segment of a leaf of $\mathcal{F}(\widetilde{\rho})$. By compactness, if we have $L \subset M$ and $\operatorname{length}(L) \in\left[\lambda^{-1}, 1\right]$, then $\operatorname{diam} \phi(L) \asymp 1$.

Now suppose $L$ has length between $\lambda^{n-1}$ and $\lambda^{n}, n \gg 0$. Then the length of $\tilde{f}^{n}(L)$ is between $\lambda^{-1}$ and 1 . Using the action of $\mathbb{Z}^{3}$, we can assume $\widetilde{f}^{n}(L) \subset M$; then by (5.2) we have

$$
\operatorname{diam}(\widetilde{\pi}(L)) \asymp \operatorname{diam}(\phi(L))=\left|\lambda^{\prime}\right|^{-n} \operatorname{diam}\left(\phi\left(\tilde{f}^{n}(L)\right)\right) \asymp \lambda^{n / 2} .
$$

Interval exchange. Note that $\alpha=1 / \lambda$ satisfies

$$
\alpha+\alpha^{2}+\alpha^{3}=1 \text {. }
$$

Let $F:[0,1] \rightarrow[0,1]$ be the interval exchange map constructed cutting $[0,1]$ into pieces of lengths $\alpha, \alpha^{2}$ and $\alpha^{3}$, flipping each one, and then flipping [0,1] as a whole. (Here an interval is flipped by cutting it into two equal pieces, and then swapping them by translations.)

As shown in $[\mathrm{AY}]$, there is a transversal $\tau$ to $\mathcal{F}(\sigma)$ such that $(\tau, \rho \mid \tau) \cong$ $([0,1], d x)$ and the first return map to $\tau$ is given by $F$.

Consider any leaf $L(t)$ of $\mathcal{F}(\sigma)$, and let $\left\langle t_{n}\right\rangle$ denote the moments when $L\left(t_{n}\right) \in \tau$. Suppose $L\left(t_{0}\right)$ corresponds to $x \in[0,1]$; then $L\left(t_{n}\right)$ corresponds 
to $F^{n}(x)$. For each $n$ we have a natural cycle $C_{n} \in H_{1}(X, \mathbb{Z})$ obtained by joining together the endpoints of $L\left[t_{0}, t_{n}\right]$ with a segment of $\tau$. Since $\rho \mid L=0$, we have

$$
\int_{C_{n}} \rho=F^{n}(x)-x \in K \cong H_{1}(E, \mathbb{Q}) .
$$

It follows that the image of $L(t)$ under $\phi$ is well-approximated by the deterministic walk

$$
z_{n}=\left(F^{n}(x)-x\right)^{\prime} \in \mathbb{C},
$$

$n \in \mathbb{Z}$; that is,

$$
z_{n}=\phi\left(L\left(t_{n}\right)\right)+O(1) .
$$

The top image in Figure 5 shows a path in $\mathbb{C}$ with vertices $z_{n}, 0 \leq n \leq 3000$, for a typical $x \in[0,1]$. By Proposition 5.1, we have

$$
\max _{0 \leq n \leq N}\left|z_{n}\right| \asymp \sqrt{N}
$$

For more on this example, see e.g. [LPV], [HLM] and [ABB].

II. Totally real cubic example. This example was provided by E. Lanneau. The decorated $A_{6}$ Coxeter diagram

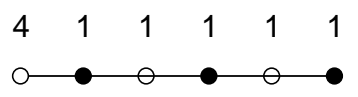

describes a configuration of six weighted simple closed curves on a surface of genus 3. The leading eigenvalue $\mu$ for their intersection matrix satisfies $\mu^{2}=\alpha$, where $\alpha=5.3234 \ldots$ is the largest root of

$$
x^{3}-8 x^{2}+15 x-4=0 .
$$

As described in $[\mathrm{Th}]$ and $[\mathrm{Mc} 4, \S 4]$, the diagram above determines a holomorphic 1-form $(X, \omega)$ of genus three and a pair of Dehn twists $\tau_{i}: X \rightarrow X$, with derivatives $D \tau_{1}=\left(\begin{array}{ll}1 & \mu \\ 0 & 1\end{array}\right)$ and $D \tau_{2}=\left(\begin{array}{ll}1 & 0 \\ \mu & 1\end{array}\right)$ in coordinates where $\omega=d z$.

The product of these Dehn twists gives a pseudo-Anosov map $f: X \rightarrow$ $X$, whose expansion factor $\lambda>1$ satisfies

$$
\lambda+\lambda^{-1}=\operatorname{tr} D f=2+\mu^{2}=2+\alpha .
$$

It turns out that $\lambda$ is still cubic over $\mathbb{Q}$; in fact, $\lambda$ is the largest root of

$$
Q(t)=t^{3}-8 t^{2}+6 t-1 .
$$


The other two roots, $\lambda^{\prime}$ and $\lambda^{\prime \prime}$, lie in $(0,1)$. We have $\lambda=(\alpha-1)(\alpha-2) / 2$.

There is a unique harmonic eigenform up to scale such that $f^{*} \rho=\lambda \rho$; indeed, $\rho$ is a linear combination of the real and imaginary parts of $\omega$. The map $f$ satisfies (5.1), and therefore $\mathcal{F}(\rho)$ has zero flux. As in the previous example, we can construct a map

$$
\phi: \widetilde{X} \rightarrow \mathbb{R}^{2}
$$

such that

$$
\phi(\widetilde{f}(x))=\left(\lambda^{\prime} \phi_{1}(x), \lambda^{\prime \prime} \phi_{2}(x)\right) .
$$

The exponent $1 / 2$ appearing in Proposition 5.1 is now replaced by two exponents satisfying $\alpha_{1}+\alpha_{2}=1$, namely $\alpha_{1}=\left|\log \lambda^{\prime}\right| / \log \lambda$ and $\alpha^{2}=$ $\left|\log \lambda^{\prime \prime}\right| / \log \lambda$, and we have:

Proposition 5.2 All sufficiently long segments of the leaves of $\mathcal{F}(\widetilde{\rho})$ satisfy

$$
\operatorname{diam}\left(\phi_{i}(L)\right) \asymp \operatorname{length}(L)^{\alpha_{i}}, \quad i=1,2 .
$$

Interval exchange. Lanneau shows an associated interval exchange is given by $F(x)=x+t_{i}$ on six consecutive intervals of length $L_{i}$, where the lengths and translations are given, as column vectors in $\mathbb{Q} \oplus \mathbb{Q} \propto \oplus \mathbb{Q} \alpha^{2}$, by

$$
\begin{aligned}
\left(L_{i}\right) & =\left(\begin{array}{cccccc}
-\frac{5}{2} & \frac{9}{4} & -\frac{19}{8} & \frac{65}{8} & -\frac{43}{8} & \frac{7}{8} \\
\frac{1}{2} & 1 & -\frac{3}{2} & -\frac{23}{2} & 7 & \frac{9}{2} \\
0 & -\frac{1}{4} & \frac{3}{8} & \frac{15}{8} & -\frac{9}{8} & -\frac{7}{8}
\end{array}\right), \\
\left(t_{i}\right) & =\left(\begin{array}{ccccccc}
\frac{3}{4} & \frac{1}{8} & \frac{1}{4} & -\frac{9}{2} & -\frac{29}{4} & -\frac{1}{4} \\
4 & -2 & -\frac{3}{2} & \frac{23}{2} & 16 & 4 \\
-\frac{3}{4} & \frac{3}{8} & \frac{1}{4} & -2 & -\frac{11}{4} & -\frac{3}{4}
\end{array}\right) .
\end{aligned}
$$

(For example, $L_{1}=-5 / 2+\alpha / 2$.)

The associated deterministic walk in $\mathbb{R}^{2}$ is defined by $z_{n}=\left(x_{n}^{\prime}, x_{n}^{\prime \prime}\right)$, where $x \in[0,1]$ and $x_{n}=F^{n}(x)-x$. By the preceding Proposition, we have

$$
\max _{|n|<N}\left|x_{n}^{\prime}\right| \asymp N^{\alpha_{1}} \text { and } \max _{|n|<N}\left|x_{n}^{\prime \prime}\right| \asymp N^{\alpha_{2}} \text {. }
$$

The lower image in Figure 5 shows a typical walk $z_{n}$ with $0 \leq n \leq 1000$. The axes have been rescaled by different factors, to account for the fact that the coordinates grow at different rates.

Properties of the walks. In both cases these walks pass at most once through each point of a discrete set $\Lambda$, corresponding to the points of 
$H^{1}(X, \mathbb{Z})_{\rho}$ that can be represented by $x \in K \cap[-1,1]$. The walks can omit large regions in $\Lambda$, but must fill a set of positive density. Although $\left|z_{n}\right| \rightarrow \infty$, it may not do so at a predictable rate. It would be interesting to study the behavior of these walks as the relative periods of $\rho$ are varied. For example, a small twist of the Arnoux-Yoccoz example yields the periodic walk shown below.

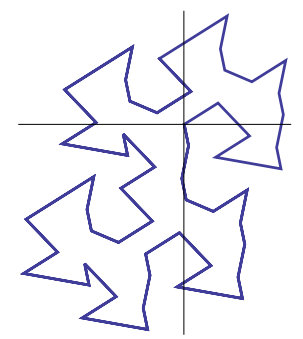

Remarks. Propositions 5.1 and 5.2 also follow from the general approach to the deviation spectrum of a measured foliation in $H^{1}(X, \mathbb{R})$ via Lyapunov exponents given in [Z]. For additional pseudo-Anosov foliations with zero flux, see [AS] and [Dy, Prop. 3]. The related theory of self-affine and selfsimilar tilings is discussed in [Ken] and [KSo].

\section{Foliations of rank two}

In this section we investigate the special properties of rank two foliations.

Theorem 6.1 If $\operatorname{deg}(X, \rho)=0$, then either $(X, \rho)$ is periodic or it has at least two minimal components.

Corollary 6.2 There is no minimal foliation of degree zero.

More precisely, we will show the ergodic factors of a minimal component of a rank two foliation all circulate in the same direction.

Theorem 6.3 Let $A \subset X_{i}$ be a saturated set of positive measure inside a minimal component of a rank two foliation. Then $\operatorname{deg}(A, \rho) \operatorname{deg}\left(X_{i}, \rho\right)>0$.

Corollary 6.4 We have $\operatorname{deg}^{+}(X, \rho)=\sum^{\prime} \operatorname{deg}\left(X_{i}, \rho\right)$, where the sum is over the minimal components of positive degree. In particular, $\operatorname{deg}^{+}(X, \rho)$ is an integer.

Proof. By the preceding result, $\operatorname{deg}(A, \rho)=\sum \operatorname{deg}\left(A \cap X_{i}, \rho\right)$ is maximized by taking $A=X_{i}$ if $\operatorname{deg}\left(X_{i}, \rho\right)>0$ and $A=\emptyset$ otherwise. 
Proof of Theorem 6.1. If $X_{i}$ is a minimal component, then $\operatorname{deg}\left(X_{i}, \rho\right) \neq 0$ by Theorem 6.3. Since $\operatorname{deg}(X, \rho)=\sum_{1}^{s} \operatorname{deg}\left(X_{i}, \rho\right)$, the result follows.

Proof of Theorem 6.3. Let $\rho=a_{1} \xi_{1}+a_{2} \xi_{2}$ as usual. The limits

$$
f_{i}(x)=\lim _{T \rightarrow \infty} \frac{1}{T} \int_{\mathcal{L}(x, T)} \xi_{i}
$$

$i=1,2$ exist a.e. and satisfy $a_{1} f_{1}(x)+a_{2} f_{2}(x)=0$. Moreover, by Theorem 4.5 , we have

$$
\left\langle\operatorname{flux}(A, \rho), \xi_{1}\right\rangle=\int_{A} f_{1}(x)|\omega|^{2}=\int_{A} \rho \wedge \xi_{1}=-a_{2} \operatorname{deg}(A, \rho)
$$

for every saturated set $A$.

Since $\left(X_{i}, \rho\right)$ is minimal, we have $\left|f_{1}(x)\right|>0$ for a.e. $x \in X_{i}$, by Corollary 4.4. If $f_{1}(x)>0$ a.e., then its integral is positive over any set $A \subset X_{i}$ of positive measure, and hence $\operatorname{deg}(A, \rho) \operatorname{deg}\left(X_{i}, \rho\right)>0$ by (6.1). The same is true if $f_{1}(x)<0$ a.e.

Now suppose $f_{1}(a)>0$ and $f_{1}(b)<0$ for some $a, b \in X_{i}$. Since $f_{1}(a)>0$, the set

$$
U_{+}=\left\{x \in X_{i}: \limsup _{T \rightarrow \infty} \int_{\mathcal{L}(x, T)} \xi_{1}=+\infty\right\}
$$

is a nonempty, saturated $G_{\delta}$. By minimality, it is also dense in $X_{i}$. Since $f_{1}(b)<0$, the same is true of

$$
U_{-}=\left\{x \in X_{i}: \liminf _{T \rightarrow \infty} \int_{\mathcal{L}(x, T)} \xi_{1}=-\infty\right\}
$$

(Both sets exclude the finitely leaves through the zeros of $\rho$.)

Since $X_{i}$ is compact, there exists a point $c \in U_{+} \cap U_{-}$. Consider any parameterized leaf $L: \mathbb{R} \rightarrow \widetilde{X}$ lying over $c \in X$ as in $\S 4$, and let $\tilde{\pi}(L(t))=$ $\left(x_{1}(t), x_{2}(t)\right) \in \mathbb{R}^{2}$. Since $\xi_{1}=d x_{1}$, the coordinate $x_{1}(t)$ accumulates on both $+\infty$ and $-\infty$ as $t \rightarrow \infty$, and hence $x_{1}(t)$ returns infinitely often to zero. Since $a_{1} x_{1}(t)+a_{2} x_{2}(t)$ is constant, and $\widetilde{\pi}$ is proper, the leaf $L(t)$ itself is recurrent. But then $L(t)$ is periodic by Theorem 4.1, contrary to our assumption that $\left(X_{i}, \rho\right)$ is minimal. 
Behavior of degree. Finally we note that Corollary 2.2 implies:

Theorem 6.5 Let $\left(X_{t}, \rho_{t}\right)$ be a continuous family of forms of rank two with constant absolute periods. Then

$$
\limsup _{t \rightarrow a} \operatorname{deg}^{+}\left(X_{t}, \rho_{t}\right) \leq \operatorname{deg}^{+}\left(X_{a}, \rho_{a}\right) .
$$

One of our main goals is to sharpen this result to a strict inequality under suitable conditions; see $\S 8$.

Remark. Theorem 6.1 strengthens [Ar1, Thm 3.7], which assumes $\mathcal{F}(\rho)$ is ergodic. I am grateful to P. Arnoux for this reference. For a related result, see [Mc2, Thm. 2.1].

Remark: Masur-Veech examples. A minimal foliation of rank two need not be uniquely ergodic. Indeed, Veech and Masur gave examples of non-ergodic minimal foliations in genus two which arise as 2-fold branched covers of a foliated torus, and hence have rank two; see e.g. [MT, §3]. Unique ergodicity does follow if the relative periods of $\rho$ have rank two [Bo, Prop. 3.8], [Mc7, Thm. 2.5]. (Note that the definition of rank in [Bo] is different from ours.)

\section{Genus two}

In this section we discuss harmonic forms of genus two. We will first show:

Theorem 7.1 Let $(X, \rho)$ be a form of genus two with $\operatorname{deg}(X, \rho)=0$. Then either $(X, \rho)$ is periodic, or it splits into two minimal components, each of genus one.

Compare [Mc2, Theorem 7.1]. The case of two minimal components is conveniently described in the language of connected sums, as we elaborate below. We then discuss topological equivalence of connected sums, and show:

Proposition 7.2 Let $v:[-1,1] \rightarrow \mathbb{C}$ be a bounded function with $\operatorname{Re} v(t)=$ t. Then for all $t$ sufficiently small, the topological type

$$
\left(X_{t}, \rho_{t}\right)=\left(E_{1}, \sigma_{1}\right) \underset{[0, v(t)]}{\#}\left(E_{2}, \sigma_{2}\right)
$$

depends only on $t$ and the topological type of $\left(E_{1}, \sigma_{1}\right)$ and $\left(E_{2}, \sigma_{2}\right)$. 
Note we do not assume that $v$ is continuous, nor do we require that $\operatorname{deg}\left(X_{0}, \rho_{0}\right)=$ 0 .

Connected sums. Here is a useful description of forms of genus two (for details see $[\mathrm{Mc5}, \S 7])$. Let

$$
\left(E_{i}, \rho_{i}\right)=\left(\mathbb{C} / \Lambda_{i}, d x\right)
$$

be a pair of harmonic forms of genus one, and let $I=[0, v]=[0,1] \cdot v$ be the segment from 0 to $v \neq 0$ in $\mathbb{C}$. Assume the projection $I \rightarrow E_{i}$ is an embedding for $i=1,2$. We can then slit $E_{1}$ and $E_{2}$ open along the image of $I$, and glue the corresponding edges, to obtain the connected sum

$$
(X, \rho)=\left(E_{1}, \rho_{1}\right) \underset{I}{\#}\left(E_{2}, \rho_{2}\right) .
$$

Every form of genus two with distinct zeros can be obtained in this way.

If $I=[0, i y]$ is vertical, then it results in a pair of saddle connections $J, J^{\prime}$ for $\mathcal{F}(\sigma)$ which join the zeros $Z(\rho)$. These arcs are exchanged by the hyperelliptic involution $\eta$ of $X$; conversely, any pair of saddle connections swapped by $\eta$ split $(X, \rho)$ into a connected sum of tori.

If $I$ is vertical and each summand $\left(E_{i}, \rho_{i}\right)$ is minimal, then $(X, \rho)$ has two minimal components, one for each torus. Conversely, if a form $(X, \rho)$ of genus two has two minimal components, then it splits canonically as a connected sum.

Relative and absolute periods. Let $(X, \rho)$ be a form of genus two with distinct zeros $a, b$. The absolute periods of $\rho$ are given by the class $[\rho] \in$ $H^{1}(X)$. The relative periods

$$
[\rho] \in H^{1}(X, Z(\rho))
$$

contain the additional inform of the quantity

$$
t=\int_{a}^{b} \rho
$$

The value of $t$ depends on the choice of a path joining the zeros.

For a connected sum, the interval $I=[0, v]$ furnishes a natural path that gives $t=\operatorname{Re} v$. The absolute periods are independent of $I$.

Proof of Theorem 7.1. Suppose $(X, \rho)$ is not periodic. Then it has at least two minimal components $X_{1}$ and $X_{2}$, each of genus 1 by (3.1). Moreover

$$
-2=\chi(X)=\chi(B)+\sum_{1}^{s} \chi\left(\operatorname{int} X_{i}\right) \leq \chi\left(\operatorname{int} X_{1}\right)+\chi\left(\operatorname{int} X_{2}\right)
$$


where $B=\bigcup \partial X_{i}$. Thus $\chi\left(\operatorname{int} X_{i}\right)=-1$ for $i=1,2$ and $\chi(B)=0$. It follows that $X_{i} \subset X$ is an embedded torus with one boundary component $B_{i}$ for $i=1,2$.

Since $\left[B_{i}\right]=0$ in $H^{1}(X)$, we have $\int_{B_{i}} * \rho=0$ for $i=1,2$, and hence $B_{i}$ must include both zeros of $\rho$. (Here we have used the fact that $\rho$ is harmonic.) Thus $B_{1}=B_{2}$, and hence $X=X_{1} \cup X_{2}$.

Corollary 7.3 A form of genus two with $\operatorname{deg}(X, \rho)=\operatorname{deg}^{+}(X, \rho)=0$ is periodic.

Proof. When $X=X_{1} \cup X_{2}$ decomposes into a pair of minimal tori, each one is ergodic; hence $\operatorname{deg}^{+}(X, \rho)=\left|\operatorname{deg}\left(X_{i}, \rho\right)\right|$ for $i=1,2$; and $\operatorname{deg}\left(X_{i}, \rho\right) \neq 0$ because $\operatorname{deg}\left(E_{i}, \rho_{i}\right) \neq 0$.

Corollary 7.4 A form of genus two with $\operatorname{deg}(X, \rho)=0$ and $|Z(\rho)|=1$ is periodic.

Proof. The foliation $\mathcal{F}(\rho)$ cannot have two minimal components of genus one, because its saddle connections form a bouquet of circles.

Isomorphic transversals. Let $\operatorname{Aut}_{0}(X, \rho)$ denote the group of smooth maps $f: X \rightarrow X$, isotopic to the identity, such that $f^{*}(\rho)=\rho$.

For cut and paste constructions like the connected sum, it is useful to know when two oriented transversals $\alpha$ and $\beta$ to $\mathcal{F}(\rho)$ are related by an automorphism of $(X, \rho)$. Clearly we must have $\int_{\alpha} \rho=\int_{\beta} \rho$, but this condition is not sufficient. Here is an analysis for linear transversals on a torus.

Proposition 7.5 Let $(E, \rho)=(\mathbb{C} / \Lambda, d x)$, and let $[0, A],[0, B] \subset \mathbb{C}$ define embedded arcs $\alpha, \beta \subset E$ transverse to $\mathcal{F}(\rho)$. Then $\alpha=f(\beta)$ for some $f \in \operatorname{Aut}_{0}(E, \rho)$ iff $\operatorname{Re}(A)=\operatorname{Re}(B)$ and the triangle $\Delta(0, A, B) \subset \mathbb{C}$ meets $\Lambda$ only at $z=0$.

Proof. When the conditions hold, $\alpha_{t}=[0,(1-t) A+t B]$ gives a family of transversals joining $\alpha$ to $\beta$. Nearby transversals in this family are related by flowing along the leaves of $\mathcal{F}(\rho)$, so they are all are equivalent under the action of $\operatorname{Aut}_{0}(E, \rho)$.

Conversely, suppose $\alpha=f(\beta)$. Then $\operatorname{Re}(A)=\int_{\alpha} \rho=\int_{\beta} \rho=\operatorname{Re}(B)$. Let $L(\alpha)$ denote set of the loops in $\pi_{1}(E, 0)$ that can be obtained by first 
running positively along $\alpha$ to a point $p \in \alpha$, and then returning to the origin by running positively along the leaf of $\mathcal{F}(\rho)$ through $p$. Geometrically, $L(\alpha)$ just consists of the points of $\Lambda \cong \pi_{1}(E)$ lying above the segment $[0, A]$. Since $f$ induces the identity on $\pi_{1}(E)$, we have $L(\alpha)=L(\beta)$. Thus there can be no lattice points in the triangle with vertices $(0, A, B)$.

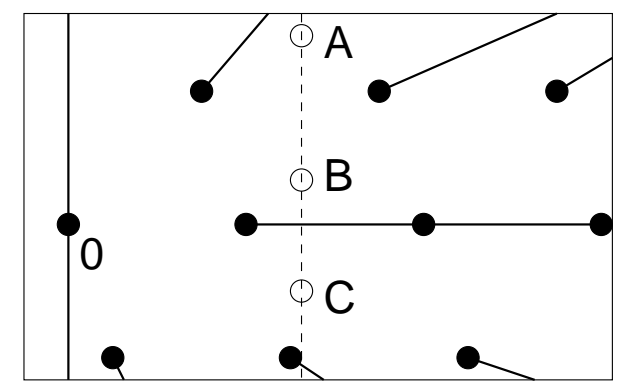

Figure 6. The picture shows $\mathbb{C}$ with the rays through $\Lambda-\{0\}$ and the line $x=0$ removed. Any remaining point $z$ determines an embedded arc $[0, z] \subset E=\mathbb{C} / \Lambda$, transverse to the foliation of $E$ by vertical lines. The transversals $[0, A]$ and $[0, B]$ are equivalent to each other, but not to $[0, C]$.

Topological equivalence. The harmonic forms $(X, \rho)$ and $\left(X^{\prime}, \rho^{\prime}\right)$ have the same topological type if there is an orientation-preserving homeomorphism $f: X \rightarrow X^{\prime}$ that sends $\mathcal{F}(\rho)$ to $\mathcal{F}\left(\rho^{\prime}\right)$ as a measured foliation. In this case we write

$$
(X, \rho) \approx\left(X^{\prime}, \rho^{\prime}\right) .
$$

Forms of the same type have the same topological and measurable dynamics, and their homological invariants agree under the isomorphism $f_{*}: H^{1}(X) \rightarrow$ $H^{1}\left(X^{\prime}\right)$.

Genus one and two. For example, two irrational foliations in genus one have the same topological type iff they have the same periods and the map

$$
H^{1}(X, \mathbb{Z}) \cong \operatorname{Per}(\rho)=\operatorname{Per}\left(\rho^{\prime}\right) \cong H^{1}\left(X^{\prime}, \mathbb{Z}\right)
$$

preserves orientation. In this case there is a unique real-linear map $\pi: X \rightarrow$ $X^{\prime}$ such that $\pi^{*}\left(\rho^{\prime}\right)=\rho$. Similarly, we note that

$$
\underset{[0, A]}{\#}\left(E_{1}, \rho_{1}\right) \underset{[0, B]}{\#}\left(E_{2}, \rho_{2}\right) \approx\left(E_{1}, \rho_{1}\right)
$$


so long as $\operatorname{Re}(A)=\operatorname{Re}(B)$ and $\Delta(0, A, B)$ embeds into both $E_{1}$ and $E_{2}$.

Stable curves. It is useful to extend the definition of connected sum to the case $I=[0]$ by defining

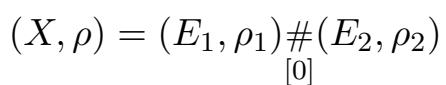

so that $X$ is the stable nodal curve $E_{1} \vee E_{2}$ obtained by identifying the two summands at a single point, and $\rho \mid E_{i}=\rho_{i}$ for $i=1,2$. The definitions of degree and flux extend naturally to these forms as well.

It is similarly useful to broaden the notion of topological equivalence so that

$$
\underset{[0]}{\left(E_{1}, \rho_{1}\right) \underset{[i y]}{\#}\left(E_{2}, \rho_{2}\right)} \approx\left(E_{1}, \rho_{1}\right) \underset{[i y}{\#}\left(E_{2}, \rho_{2}\right)
$$

for all $y \neq 0$. In other words, we allow the collapse of a pair of saddle connection to a node. The invariants of a form are also preserved by this broader relation.

With this convention, we may allow $y(0)=0$ in Proposition 7.2.

Proof of Proposition 7.2. Consider a second family of forms

$$
\left(X_{t}^{\prime}, \rho_{t}^{\prime}\right)=\left(E_{1}^{\prime}, \sigma_{1}^{\prime}\right) \underset{\left[0, v^{\prime}(t)\right]}{\#}\left(E_{2}^{\prime}, \sigma_{2}^{\prime}\right),
$$

satisfying $\left(E_{i}^{\prime}, \rho_{i}^{\prime}\right) \approx\left(E_{i}, \rho_{i}\right)$ for $i=1,2$ and $\operatorname{Re} v^{\prime}(t)=t$. Let $\pi_{i}: E_{i}^{\prime} \rightarrow E_{i}$ be real linear maps transporting $\rho_{i}^{\prime}$ to $\rho_{i}$ for $i=1,2$, and let $w_{i}(t)=\pi_{i}\left(v^{\prime}(t)\right)$. Then $\left(X_{t}^{\prime}, \rho_{t}^{\prime}\right)$ can be constructed, up to topological equivalence, by cutting $\left(E_{i}, \rho_{i}\right)$ open along $\left[0, w_{i}(t)\right]$ and gluing the slits together.

Note that $\operatorname{Re} w_{i}(t)=t$ and $|v(t)|$ and $\left|w_{i}(t)\right|$ are uniformly bounded. Thus for $t \neq 0$ sufficiently small, Proposition 7.5 guarantees that the transversals $[0, v(t)]$ and $\left[0, w_{i}(t)\right]$ are equivalent under $\operatorname{Aut}_{0}\left(E_{i}, \sigma_{i}\right), i=1,2$. It follows that $\left(X_{t}^{\prime}, \rho_{t}^{\prime}\right) \approx\left(X_{t}, \rho_{t}\right)$.

The case $t=0$ is immediate, provided we use the convention above on stable curves when $v(0)=0$.

Corollary 7.6 Let $\left(X_{t}, \rho_{t}\right)$ be a continuous family of harmonic forms of genus two with constant absolute periods and relative period $t$. Then there exist lattices such that

$$
\left(X_{t}, \rho_{t}\right) \approx\left(\mathbb{C} / \Lambda_{1}, d x\right) \#\left(\mathbb{C} / \Lambda_{2}, d x\right)
$$

for all t sufficiently small. 
Proof. Use the fact that the period map is a local homeomorphism to write $\left(X_{t}, \rho_{t}\right)$ as a continuously varying connected sum, and then apply Proposition 7.2.

\section{Bifurcations and self-similarity}

Let $\Lambda_{1}, \Lambda_{2}$ be lattices in $\mathbb{C}$. In this section we study the bifurcation locus in the family of harmonic forms of genus two defined by

$$
\left(X_{t}, \rho_{t}\right)=\left(\mathbb{C} / \Lambda_{1}, d x\right) \underset{[0, t]}{\#}\left(\mathbb{C} / \Lambda_{2}, d x\right) .
$$

Using this analysis, we establish Theorems 1.2, 1.3 and 1.4.

Bifurcation locus. We assume throughout this section that absolute periods of $\rho_{0}$ have rank two, and $\operatorname{deg}\left(X_{0}, \rho_{0}\right)=0$. The family (8.1) is defined for all $t$ in the open interval $(-a, a)$, where

$$
a=\inf \left\{x>0: x \in \Lambda_{1} \cup \Lambda_{2}\right\} .
$$

(We allow $a=\infty$.) Note that $\left(X_{0}, \rho_{0}\right)$ is actually a form on a stable curve. The absolute periods of $\rho_{t}$ are constant, and therefore

$$
\operatorname{deg}\left(X_{t}, \rho_{t}\right)=0
$$

for all $t$.

The bifurcation locus is defined by

$$
B=B\left(\Lambda_{1}, \Lambda_{2}\right)=\left\{t \in(-a, a):\left(X_{t}, \rho_{t}\right) \text { is not periodic }\right\} .
$$

Note that $\left(X_{t}, \rho_{t}\right)$ is isomorphic to $\left(X_{-t}, \rho_{-t}\right)$, and thus $B=-B$.

Theorem 8.1 The bifurcation locus is a closed, countable subset of $(-a, a)$.

Proof. By Theorem 7.1, for any $t \in(-a, a)$ the foliation $\mathcal{F}\left(\rho_{t}\right)$ is either periodic (and $t \notin B$ ), or it consists of two minimal components (and $t \in B$ ).

In the first case $\mathcal{F}\left(\rho_{t}\right)$ has at least one cylinder. Since the absolute periods of $\rho_{t}$ are fixed, this cylinder persists under small deformations, and therefore $B$ is closed. In the second case there is a saddle connection $J$ joining the zeros of $\rho_{t}$. Since $\int_{J} \rho_{t}=0$, we must have $t \in \operatorname{Per}\left(\rho_{t}\right)=\operatorname{Per}\left(\rho_{0}\right)$, and hence $B$ is countable. 
Theorem 8.2 For any $b \in B$, we have

$$
\limsup _{t \rightarrow b} \operatorname{deg}^{+}\left(X_{t}, \rho_{t}\right) \leq \operatorname{deg}^{+}\left(X_{b}, \rho_{b}\right)-1 .
$$

Corollary 8.3 The bifurcation locus is homeomorphic to a closed subset of $\omega^{\omega}$.

Equivalently, the derived sets

$$
B \supset B^{\prime} \supset B^{\prime \prime} \supset \cdots B^{(k)}
$$

satisfy $\bigcap B^{(k)}=\emptyset$. Here $A^{\prime}$ consists of the non-isolated points of $A$.

Using Corollary 7.4, one can show that $B \subset(-a, a)$ is compact whenever $a$ (defined by (8.2) is finite. In this case $B$ is homeomorphic to an ordinal $\xi<\omega^{k}$ for some natural number $k$. Note however that $B$ is usually not well-ordered as a subset of $\mathbb{R}$; since $B=-B$, whenever $0 \in B^{\prime}$ the origin is a limit point of $B$ from both above and below.

Theorem 8.4 If the periods $\operatorname{Per}\left(\rho_{0}\right)$ lie in a real quadratic field, then $B$ is self-similar about every point.

For example, suppose $0 \in B^{(k)}$ and $\operatorname{Per}\left(\rho_{0}\right) \subset \mathbb{Q}(\sqrt{D})$. Since $B=-B$, Theorem 8.4 implies that there exist $A_{i}>0$ and $\epsilon_{i} \in(0,1)$ such that all points of the form

$$
t= \pm A_{1} \epsilon_{1}^{n_{1}} \pm A_{2} \epsilon_{1}^{n_{1}} \epsilon_{2}^{n_{2}} \pm \cdots \pm A_{k} \epsilon_{1}^{n_{1}} \epsilon_{2}^{n_{2}} \cdots \epsilon_{k}^{n_{k}}
$$

with integral exponents $n_{i} \geq 0$, belong to $B$.

Germs of bifurcations. We begin by making Theorem 8.4 more precise. Let us say $A, B \subset \mathbb{R}$ have the same germ at zero if there is a neighborhood $U$ of the origin such that $A \cap U=B \cap U$.

We say $A$ is self-similar at $x$ if there is a $\lambda>1$ such that $(A-x)$ and $\lambda(A-x)$ have the same germ at zero. If this statement holds for all $x \in \mathbb{R}$, we say $A$ is self-similar about every point.

By Proposition 7.2 we have:

Proposition 8.5 The germs at zero of the set $B\left(\Lambda_{1}, \Lambda_{2}\right)$ and the function $\operatorname{deg}^{+}\left(X_{t}, \rho_{t}\right)$ depends only on the oriented groups $P_{i}=\operatorname{Re} \Lambda_{i} \subset \mathbb{R}, i=1,2$.

Now suppose $b \in B$. Then we have a vertical connected sum decomposition

$$
\left(X_{b}, \rho_{b}\right)=\left(\mathbb{C} / \Lambda_{1}^{\prime}, d x\right) \underset{[0, i y]}{\#}\left(\mathbb{C} / \Lambda_{2}^{\prime}, d x\right),
$$


which determines a second family of forms

$$
\left(X_{t}^{\prime}, \rho_{t}^{\prime}\right)=\left(\mathbb{C} / \Lambda_{1}^{\prime}, d x\right) \underset{[0, t]}{\#}\left(\mathbb{C} / \Lambda_{2}^{\prime}, d x\right) .
$$

Then Proposition 7.2 also implies:

Proposition 8.6 The set $B\left(\Lambda_{1}, \Lambda_{2}\right)$-b has the same germ at zero as $B\left(\Lambda_{1}^{\prime}, \Lambda_{2}^{\prime}\right)$, and

$$
\operatorname{deg}^{+}\left(X_{t}^{\prime}, \rho_{t}^{\prime}\right)=\operatorname{deg}^{+}\left(X_{b+t}, \rho_{b+t}\right)
$$

for all t sufficiently small.

Proof of Theorem 8.2. By Proposition 8.6 we may assume $b=0$. By Corollary 7.3, we have $d=\operatorname{deg}^{+}\left(X_{0}, \rho_{0}\right)>0$.

Choosing a basis, we can write $\operatorname{Per}(\rho)=\mathbb{Z} a_{1} \oplus \mathbb{Z} a_{2}=\operatorname{Re} \Lambda$, where

$$
\Lambda=\left(a_{1}-i a_{2}\right) \mathbb{Z}[i] \subset \mathbb{C} .
$$

Using Proposition 8.5, we can reduce to the case where $\Lambda_{1}$ has index $d$ in $\Lambda$ and $\Lambda_{2}$ has index $d$ in its complex conjugate, $\bar{\Lambda}$. The conjugate intervenes because $\operatorname{deg}\left(X_{0}, \rho_{0}\right)=0$.

Let $E=\mathbb{C} / \Lambda$ and let $E_{i}=\mathbb{C} / \Lambda_{i}$ for $i=1,2$. Then we have natural covering maps $f_{i}: E_{i} \rightarrow E$ of degree $(-1)^{i+1} d$, given by $f_{1}([z])=[z]$ and $f_{2}([z])=[\bar{z}]$. We also have

$$
\left(X_{t}, \rho_{t}\right)=\left(E_{1}, d x\right) \underset{[0, t]}{\#}\left(E_{2}, d x\right) .
$$

The period torus of $\left(X_{t}, \rho_{t}\right)$ can be identified with $E$, and the period map

$$
\pi_{t}: X_{t} \rightarrow E
$$

can be constructed by gluing the maps $f_{i}: E_{i} \rightarrow E$ together along the slit $[0, t]$.

Suppose $\operatorname{deg}^{+}\left(X_{t}, \rho_{t}\right)>0$. Then there is a closed subsurface $A$ of $\left(X_{t}, \rho_{t}\right)$ which maximizes $\operatorname{deg}\left(A, \rho_{t}\right)$, by Corollary 6.4. In terms of the connected sum description (8.1) of $X_{t}$, the part of $A$ inside $E_{2}$ contributes negatively to its degree, and the part inside $E_{1}$ contributes at most $\operatorname{deg}\left(f_{1}\right)=d$. For equality to be achieved, $A$ must fill $E_{1}$; but this is impossible, since $E_{1}$ does not give a saturated subset of $X_{t}$ when $t \neq 0$. (Put differently, ergodicity of the irrational foliation $\mathcal{F}\left(\sigma_{1}\right)$ forces any saturated set of positive measure to meet its transversal $[0, t]$.) Since the upper degree is an integer (Corollary 6.4), we have $\operatorname{deg}^{+}\left(X_{t}, \rho_{t}\right) \leq d-1$. 
Proof of Corollary 8.3. By a classical result [MS], the closed, countable set $B$ is homeomorphic to a countable ordinal $\xi$. Let $B_{d}=\{t$ : $\left.\operatorname{deg}^{+}\left(X_{t}, \rho_{t}\right) \geq d\right\}$. Then $B=B_{1}$ and we have $B_{d}^{\prime} \subset B_{d+1}$ by Theorem 8.2. Thus $\bigcap B^{(k)}=\bigcap B_{d}=\emptyset$. This implies $\xi \leq \omega^{\omega}$, since any larger ordinal has $\bigcap \xi^{(k)} \neq \emptyset$.

The same reasoning shows:

Corollary 8.7. If $0 \in B^{(k)}$, then $k<\operatorname{deg}^{+}\left(X_{0}, \rho_{0}\right)$.

Proof of Theorem 8.4. By assumption there is a real quadratic field $K=\mathbb{Q}(\sqrt{D})$ which contains $P_{i}=\operatorname{Re}\left(\Lambda_{i}\right)$ for $i=1,2$. Let $x \mapsto x^{\prime}$ denote the Galois involution on $K$. Using Proposition 8.5, we can reduce to the case where

$$
\Lambda_{1}=\left\{x+i x^{\prime}: x \in P_{1}\right\} \text { and } \Lambda_{2}=\left\{x-i x^{\prime}: x \in P_{2}\right\} .
$$

The reversal of sign comes about again because $\operatorname{deg}\left(X_{0}, \rho_{0}\right)=0$.

Since each $P_{i} \subset K$ is a fractional ideal for some order in $K$, there is a unit $\epsilon>1$ in $K$ stabilizing both (see e.g. [BoS, Ch. 2]). We may assume $\epsilon^{\prime}>0$ as well. Then the real-linear map

$$
f(x+i y)=\epsilon x+i \epsilon^{\prime} y
$$

satisfies $f\left(\Lambda_{i}\right)=\Lambda_{i}$ for $i=1,2$, and gives rise to a topological equivalence

$$
\left(X_{t}, \rho_{t}\right) \approx\left(X_{\epsilon t}, \rho_{\epsilon t}\right)
$$

It follows that $\epsilon B=B$, and hence $B$ is self-similar at the origin.

Proof of Theorems 1.2, 1.3 and 1.4 . The results also hold for general families of forms of genus two with fixed absolute periods, by Corollary 7.6.

\section{Coupled rotations}

In this section we relate foliations in genus two to coupled irrational rotations of the circle. We will use this relationship in the next section to give explicit bifurcation loci of high ordinal complexity. 
Coupled rotations. Consider a pair of lengths $0<L_{1} \leq L_{2}$ and twists $\tau_{1} \in\left[0, L_{1}\right), \tau_{2} \in\left[0, L_{2}\right)$. Let $X$ denote the pair of disjoint circles

$$
X=X_{1} \sqcup X_{2}=\mathbb{R} / \mathbb{Z} L_{1} \sqcup \mathbb{R} / \mathbb{Z} L_{2},
$$

and let $R: X \rightarrow X$ be the rotation given by

$$
R(x)=x+\tau_{i} \bmod L_{i} \text { if } x \in X_{i} .
$$

For $t \in\left[0, L_{1}\right)$, let $S_{t}: X \rightarrow X$ denote the interval exchange map that swaps $[0, t) \subset X_{1}$ with $[0, t) \subset X_{2}$, and otherwise acts by the identity. Let

$$
F_{t}=R \circ S_{t}: X \rightarrow X .
$$

For convenience, we extend the definition to negative $t$ so $F_{t}=F_{|t|}$.

The bifurcation set of this family is defined by

$$
B\left(L_{1}, \tau_{1}, L_{2}, \tau_{2}\right)=\left\{t \in\left(-L_{1}, L_{1}\right): F_{t} \text { is not periodic }\right\} .
$$
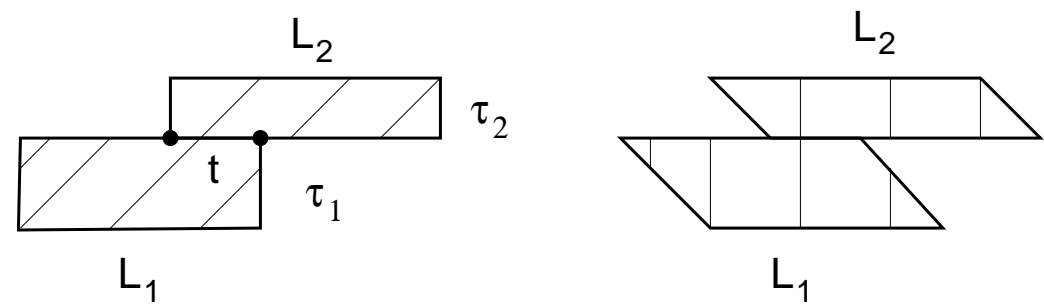

Figure 7. Foliated surfaces associated to coupled rotations.

Proposition 9.1 We have $B\left(L_{1}, \tau_{1}, L_{2}, \tau_{2}\right)=B\left(\Lambda_{1}, \Lambda_{2}\right)$, where

$$
\Lambda_{1}=\mathbb{Z} L_{1} \oplus \mathbb{Z}(1-i) \tau_{1} \quad \text { and } \quad \Lambda_{2}=\mathbb{Z} L_{2} \oplus \mathbb{Z}(1-i) \tau_{2} .
$$

Proof. Consider the diagonal foliation of a pair of stacked rectangles with dimensions $L_{i} \times \tau_{i}, i=1,2$, overlapping in length $t$. (See Figure 7.) By gluing corresponding horizontal and vertical edges, we obtain a harmonic foliation $\mathcal{F}_{t}$ on a surface of genus two. It is easy to see that $F_{t}(x)$ gives the first return of $\mathcal{F}_{t}$ to the horizontal edges of the rectangles, so periodic intervals for $F_{t}$ correspond to cylinders for $\mathcal{F}_{t}$. Making a linear change of coordinates so the foliation becomes vertical, as shown at the right, gives the lattices $\Lambda_{1}$ and $\Lambda_{2}$. 


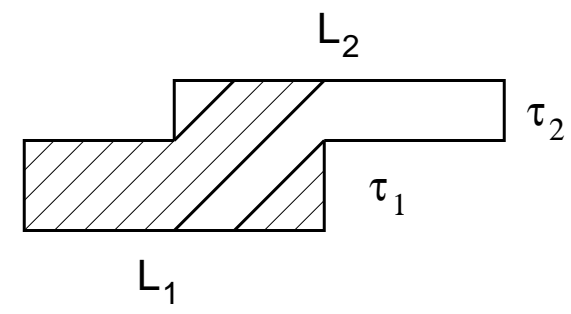

Figure 8. Splitting for $t=\tau_{1}+\tau_{2}$.

Conversely, the germ of any bifurcation set of the form $B\left(\Lambda_{1}, \Lambda_{2}\right)$ also arises as the germ of the bifurcation set for a pair of coupled rotations.

Renormalization. For certain values of $t$, one can renormalize $F_{t}$ to obtain another map of the same form. Then the new bifurcation locus reappears locally inside the old one. Here is a particular instance, which we will prove geometrically. For a more detailed discussion of this elementary move, see $[\mathrm{Mc} 3, \S 2]$.

Proposition 9.2 Suppose $L=\tau_{1}+\tau_{2}<\min \left(L_{1}, L_{2}\right)$. Then the sets $B\left(L_{1}, \tau_{1}, L_{2}, \tau_{2}\right)-L$ and $B\left(L_{1}-L, \tau_{1}, L_{2}-L, \tau_{2}\right)$ have the same germ at zero.

Proof. For $t=L$, the stacked rectangles of Figure 7 contain a square of side $L=\tau_{1}+\tau_{2}$. The diagonal of this square gives a saddle connection $J$ for the corresponding form $\left(X_{t}, \rho_{t}\right)$ (see Figure 8). Cutting along $J$ and its image under the hyperelliptic involution results in a connect sum decomposition, $\left(X_{t}, \rho_{t}\right)=\left(E_{1}, \sigma_{1}\right) \#\left(E_{2}, \sigma_{2}\right)$. The leaves lying in one summand are shown in Figure 8. Using the fact that the diagonal foliation comes from $d x-d y$, it is easy to see that $\operatorname{Per}\left(E_{i}\right)=\mathbb{Z}\left(L_{i}-t\right) \oplus \mathbb{Z} \tau_{i}$. The result now follows from Propositions 8.5, 8.6 and 9.1.

Degree zero. We define the rank and degree of $F_{t}$ to be the rank and degree of the associated foliation $\left(X_{t}, \rho_{t}\right)$. These invariants are independent of $t$. Here is a concrete characterization of degree zero:

Proposition 9.3 The coupled rotations $F_{t}$ have degree zero iff both rotations are irrational, and

$$
\left(\begin{array}{c}
L_{1} \\
\tau_{1}
\end{array}\right)=A\left(\begin{array}{l}
L_{2} \\
\tau_{2}
\end{array}\right)
$$


for some rational matrix with $\operatorname{det}(A)=-1$.

Proof. Let $P_{i}=\mathbb{Z} L_{i} \oplus \tau_{i}, i=1,2$. Then $P=\operatorname{Per}\left(\rho_{t}\right)=P_{1}+P_{2}$, and $\left(X_{t}, \rho_{t}\right)$ has degree zero iff $P$ has rank two and the subgroups $P_{i} \subset P$ have the same index but opposite orientations.

Example: positive degree. We conclude with an example illustrating some of the differences between coupled rotations of zero and non-zero degree.

Choose an irrational number $\alpha$ such that

$$
1<L_{1}=\alpha \leq L_{2}=3-\alpha
$$

and let $\theta_{1}=1$ and $\theta_{2}=-1$. Consider the family of coupled rotations $F_{t}$ determined by $\left(L_{1}, \theta_{1}, L_{2}, \theta_{2}\right)$. Since

$$
\left(\begin{array}{c}
3-\alpha \\
-1
\end{array}\right)=\left(\begin{array}{cc}
-1 & 3 \\
0 & -1
\end{array}\right)\left(\begin{array}{l}
\alpha \\
1
\end{array}\right)
$$

and the above matrix has determinant one, the maps $F_{t}$ have degree two.

Theorem 9.4 The map $F_{t}$ has an open interval of periodic points for all $t \in(1, \alpha)$, but no periodic points for $t \in[0,1)$.

Proof. It easy to see that the associated foliation $\mathcal{F}_{t}$ has a minimal component for all $t$, so no more than one cylinder is possible.

Consider an orbit of $F_{t}$ as a sequence of real numbers $x_{i}$, each lying either in $\left[0, L_{1}\right)$ or $\left[0, L_{2}\right)$. Then we have integers $A_{i}, B_{i}$ such that

$$
x_{i+1}-x_{i}=A_{i}+B_{i} \alpha .
$$

Each time the orbit passes the origin on one circle or the other, $B_{i}$ decreases by 1 . For $t<1$ this event occurs infinitely often, so there are no cylinders. On the other hand, the intervals $(1, t) \subset\left[0, L_{1}\right)$ and $(0, t) \subset\left[0, L_{2}\right)$ are interchanged by $F_{t}$ for $t>1$. Thus $F_{t}$ has an open interval of points with period two for all $t \in(1, \alpha)$.

One can also make examples that are minimal for all $t$, e.g. by coupling two copies of the same irrational rotation as in [V3]. 


\section{Quadratic examples}

This section gives explicit bifurcation sets with large ordinal complexity.

Let $\gamma=(1+\sqrt{5}) / 2$. Given $x>1$ in $\mathbb{Q}(\sqrt{5})$, let

$$
B(x)=B(x, 1, \gamma x, \gamma)
$$

be the bifurcations locus for a family of coupled rotations, as in $\S 9$. Let

$$
\delta(x)=\min \left\{k \geq 0: 0 \notin B(x)^{(k)}\right\} .
$$

By Proposition 8.5, whenever $\left(\begin{array}{ll}a & b \\ c & d\end{array}\right) \in \mathrm{SL}_{2}(\mathbb{Z})$ and $y=(a x+b) /(c x+d)>$ 0 , we have $\delta(y)=\delta(x)$. Using this invariance, we extend $\delta(x)$ to a function defined for all $x \in K \cup\{\infty\}$. Note that $\delta(x)=0$ iff $x \in \mathbb{Q} \cup\{\infty\}$.

We will show:

Theorem 10.1 For all $k>0$ we have $\delta(k \gamma)=k$.

The sets $B(k \gamma)$ for $k=1,2,3,4$ are illustrated in Figure 2. In the course of the proof we will see that the germ of $B(k \gamma)$ at $1+\gamma=2.618 \ldots$ is the same as the germ of $B((k-1) \gamma)$ at 0 , as can be seen in the Figure. In particular, the $(k-1)$ st derived set of $B(k \gamma)$ is nonempty.

Quadratic fields. The proof of Theorem 10.1 relies on two general results.

Let $K \subset \mathbb{R}$ be a real quadratic field whose ring of integers $\mathcal{O}_{K}$ has discriminant $D$. The Galois involution on $K$ will be denoted by $x \mapsto x^{\prime}$. The norm and trace are given by $N(x)=x x^{\prime}$ and $\operatorname{Tr}(x)=x+x^{\prime}$. For any $x, y \in K$ we let

$$
\operatorname{det}(x, y)=\operatorname{det}\left(\begin{array}{ll}
x & x^{\prime} \\
y & y^{\prime}
\end{array}\right) .
$$

If $x, y \in \mathcal{O}_{K}$ generate a module $J=\mathbb{Z} x \oplus \mathbb{Z} y \cong \mathbb{Z}^{2}$, then

$$
|\operatorname{det}(x, y)|=\sqrt{D}\left[\mathcal{O}_{K}: J\right] .
$$

Consider a family of coupled rotations $F_{t}(x)$ whose defining lengths $L_{1} \leq$ $L_{2}$ and twists $\tau_{1}, \tau_{2}$ belong to $K$. Assume the rotation numbers

$$
\theta_{i}=\frac{\tau_{i}}{L_{i}}
$$

are irrational. It is easy to see: 
Proposition 10.2 The coupled rotations determined by $\left(L_{1}, \tau_{1}, L_{2}, \tau_{2}\right)$ have degree zero iff

$$
\operatorname{det}\left(L_{1}, \tau_{1}\right)+\operatorname{det}\left(L_{2}, \tau_{2}\right)=0 \text {. }
$$

In this case the bifurcation locus

$$
B=B\left(L_{1}, \tau_{1}, L_{2}, \tau_{2}\right)
$$

is compact, countable and self-similar about every point, by Theorems 8.4 and Proposition 9.1. We will make this self-similarity quantitative, by showing:

Theorem 10.3 Suppose $B$ and $\epsilon B$ have the same germs at zero and $\epsilon>1$. Then

$$
(\epsilon B) \cap(-a, a)=B \cap(-a, a),
$$

where

$$
a=L_{1} \cdot \min \left(1,\left|\theta_{1}-\theta_{1}^{\prime}\right|,\left|\theta_{2}-\theta_{2}^{\prime}\right|\right)
$$

Proof. Consider the lattices

$$
\Lambda_{i}=\left\{\left(k, k^{\prime}\right): k \in \mathbb{Z} L_{i} \oplus \mathbb{Z} \tau_{i}\right\} \subset \mathbb{R}^{2} \cong \mathbb{C},
$$

$i=1,2$. As we saw in the proof of Theorem 8.4, the bifurcation locus

$$
\widetilde{B}=B\left(\Lambda_{1}, \bar{\Lambda}_{2}\right)
$$

has the same germ at zero as $B$, and satisfies $\alpha \widetilde{B}=\widetilde{B}$ for some unit $\alpha>1$ in $K$. It follows that $\epsilon \widetilde{B}=\widetilde{B}$ as well.

Let $P_{i}=\left(t, t L_{i}^{\prime} / L_{i}\right)$ and let $Q=(t, 0)$. The proof of $(10.1)$ comes down to the fact that $\left[0, P_{i}\right]$ and $[0, Q]$ are equivalent transversals to $\left(\mathbb{R}^{2} / \Lambda_{i}\right)$, for all $t$ sufficiently small. We wish to show this condition holds when $|t|<a$. By Proposition 7.5, it suffices to show that the triangle $\Delta\left(0, P_{i}, Q\right)$ contains no nonzero lattice point of $\Lambda_{i}$.

Suppose $|t|<a$. Consider the projection $\phi_{i}: \mathbb{R}^{2} \rightarrow \mathbb{R}$ given by

$$
\phi_{i}(x, y)=\operatorname{det}\left(\begin{array}{cc}
L_{i} & L_{i}^{\prime} \\
x & y
\end{array}\right) .
$$

Then $\phi_{i}(0)=\phi_{i}\left(P_{i}\right)=0$ and $\phi_{i}\left(\Lambda_{i}\right)=\mathbb{Z} \operatorname{det}\left(L_{i}, \theta_{i}\right)$. Note we have

$$
a / L_{i} \leq\left|N\left(\theta_{i}\right)\right|=\left|\operatorname{det}\left(L_{i}, \theta_{i}\right) / N\left(L_{i}\right)\right|
$$


Thus by the definition of $a$, we have

$$
\left|\phi_{i}(Q)\right|=\left|t L_{i}^{\prime}\right|=t\left|N\left(L_{i}\right) / L_{i}\right|<\left|\operatorname{det}\left(L_{i}, \theta_{i}\right)\right| .
$$

Since the triangle $\Delta\left(0, P_{i}, Q\right)$ is convex, this inequality shows it can only meet lattices points in $\operatorname{Ker}\left(\phi_{i} \mid \Lambda_{i}\right)=\mathbb{Z}\left(L_{i}, L_{i}^{\prime}\right)$. And indeed, the leg $0 Q$ of the triangle is parallel to $\left(L_{i}, L_{i}^{\prime}\right)$. But $|t|<a \leq L_{1} \leq L_{2}$, so the only lattice point in $\Delta\left(0, P_{i}, Q\right)$ is zero.

We now return to the setting of Theorem 10.1, where $K=\mathbb{Q}(\sqrt{5})$ and $\mathcal{O}_{K}=\mathbb{Z}[\gamma]$.

Theorem 10.4 For any relatively prime integers $a, b \in \mathbb{Z}[\gamma]$, we have

$$
\delta(a / b) \leq|\operatorname{det}(a, b)| / \sqrt{5} .
$$

On the other hand we have

$$
\delta(x) \geq 1+\delta(x-\gamma)
$$

provided $x$ and $\left|1-x / x^{\prime}\right|$ are both bigger than $1+\gamma$.

Proof. For the first assertion, let $P_{1}=\mathbb{Z} a \oplus \mathbb{Z} b$ and let $P_{2}=\gamma P_{1}$. Then $P=P_{1}+P_{2}$ is a module over $\mathcal{O}_{K}$. Since $a$ and $b$ are relatively prime, we have $P=\mathcal{O}_{K}$, a ring of discriminant $D=5$. Thus the family of 1 -forms determined by this data satisfies

$$
\operatorname{deg}_{+}\left(X_{0}, \rho_{0}\right)=\left|P / P_{i}\right|=|\operatorname{det}(a, b)| / \sqrt{5} .
$$

The upper bound on the depth now follows from Corollary 8.7.

For the second assertion, recall from Theorem 8.4 that $B(x)$ is self-similar at zero; that is, $B(x)$ and $\epsilon B(x)$ have the same germs for some $\epsilon>1$. (In fact we may take $\epsilon$ to be a power of $\gamma$ ).

Suppose $x>1+\gamma=\tau_{1}+\tau_{2}$. Then by Proposition 9.2, the sets $B(x)-1-\gamma$ and $B(x-1-\gamma, 1, \gamma x-1-\gamma, \gamma)$ have the same germs at zero. By Proposition 7.2 , the latter set has the same germ as

$$
B(x-\gamma, 1, \gamma x-1, \gamma)
$$

which in turn has the same germ as $B(x-\gamma)$, using the fact that $\gamma^{2}=\gamma+1$. Consequently we have

$$
1+\gamma \in B(x)^{(k-1)},
$$


where $k=\delta(x-\gamma)$.

Now suppose in addition that $\left|1-x / x^{\prime}\right|>1+\gamma$. Then by Theorem 10.3 $B(x)$ is self-similar on the interval $(-a, a)$, where

$$
a=L_{1} \min \left(1,\left|\theta_{1}-\theta_{1}^{\prime}\right|\right)=\min \left(x,\left|1-x / x^{\prime}\right|\right)>1+\gamma .
$$

Consequently the sequence $\epsilon^{-i}(1+\gamma)$ lies in $B(x)^{(k)}$ as well, for all $i>0$. Therefore $0 \in B(x)^{(k+1)}$ and hence $\delta(x)>1+k=1+\delta(x-\gamma)$.

Proof of Theorem 10.1. Clearly $\delta(\gamma) \geq 1$. For $x=k \gamma$ with $k>1$, we have $x>1+\gamma$ and $\left|1-x / x^{\prime}\right|=1+\gamma^{2}>1+\gamma$, so $\delta(k \gamma) \geq k$ by induction and (10.3). On the other hand, $|\operatorname{det}(k \gamma, 1)|=k\left|\gamma-\gamma^{\prime}\right|=k \sqrt{5}$, so $\delta(k \gamma) \leq k$ by $(10.2)$.

The proof shows $t=0$ is the only point in $B(k \gamma)^{(k-1)}$ with $|t|<1+\gamma$.

\section{A The periodic foliations of a Teichmüller curve}

Let $(X, \omega)$ be a nonzero holomorphic 1-form of genus $g$. Geodesics on the (singular) flat surface $(X,|\omega|)$ fall into parallel families which are described by the foliations $\mathcal{F}\left(\rho_{t}\right)$, where $\rho_{t}=\operatorname{Re}\left(e^{i t} \omega\right)$.

In this section we will show that the content $C\left(\rho_{t}\right) \subset H^{1}(X)$ can be readily determined whenever $(X, \omega)$ generates a Teichmüller curve. This invariant provides a geometric picture, at the level of cohomology, of the fluctuating foliation $\mathcal{F}\left(\rho_{t}\right)$.

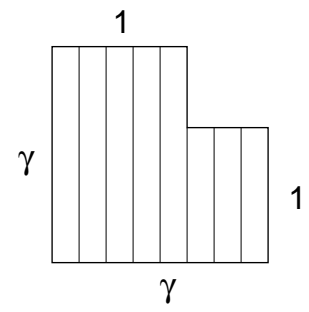

Figure 9. Gluing opposite edges results in a surface of genus two.

The golden table. As an illustrative example, let $D=5$, let $\gamma=(1+\sqrt{5}) / 2$ be the golden mean, and let $\mathcal{O}_{D}=\mathbb{Z}[\gamma]$ be the ring of integers in the field

$$
K=\mathbb{Q}(\sqrt{5}) \subset \mathbb{R} .
$$


The Galois involution of $K / \mathbb{Q}$ will be denoted by $x \mapsto x^{\prime}$. We will see below that every $x \in \mathbb{P}^{1}(K)$ has a unique expression as a canonical fraction $x=a / b, a, b \in \mathcal{O}_{D}$.

Let $(X, \omega)=(P, d z) / \sim$ be the holomorphic 1-form of genus two obtained from the $L$-shaped region $P \subset \mathbb{C}$ shown in Figure 9 by gluing opposite sides. It is well-known that

$$
\mathrm{SL}(X, \omega)=\left\langle\left(\begin{array}{ll}
1 & \gamma \\
0 & 1
\end{array}\right),\left(\begin{array}{cc}
0 & 1 \\
-1 & 0
\end{array}\right)\right\rangle \subset \mathrm{SL}_{2}(\mathbb{R})
$$

is the $(2,5, \infty)$ triangle group, and hence $(X, \omega)$ generates a Teichmüller curve $V \rightarrow \mathcal{M}_{2}$. This curve is closely related to billiards in the regular pentagon [V2], [Mc1, §9].

The foliation $\mathcal{F}\left(\rho_{t}\right)$ is periodic when $\tan (t) \in K \cup\{\infty\}$; otherwise, it is uniquely ergodic. In the uniquely ergodic case, $C\left(\rho_{t}\right)$ is simply the segment joining 0 to $\left[\rho_{t}\right]$ in $H^{1}(X)$; that is,

$$
C\left(\rho_{t}\right)=\left[0, \rho_{t}\right] \text { when } t \notin K .
$$

The periodic case is handled by the following result.

Theorem A.1 Let $\tan (t)=a / b$ be a canonical fraction in $K$, with $|t|<$ $\pi / 2$, let $A_{t} \in H^{1}(X, \mathbb{Z})$ be the unique integral class satisfying

$$
\int A_{t} \wedge \omega=a+i b
$$

and let $B_{t}=A_{t} / \sqrt{a^{2}+b^{2}}$. Then the content of $\rho_{t}$ is the parallelogram in $H^{1}(X)$ given by

$$
C\left(\rho_{t}\right)=\left\{(x-y) B_{t} / \gamma+y\left[\rho_{t}\right]:(x, y) \in[0,1]^{2}\right\} .
$$

See Figure 10. Geometrically, $A_{t}$ is simply the Poincaré dual of one of the closed leaves of $\mathcal{F}\left(\rho_{t}\right)$.

Galois conjugates. To describe the behavior of the normalized class $B_{t}$ in more detail, we remark that the map $C \mapsto \int_{C} \omega$ gives an isomorphism

$$
H_{1}(X, \mathbb{Z}) \cong \mathcal{O}_{D}[i] \text {. }
$$

There is a second holomorphic form $\omega^{\prime}$ on $X$ which satisfies

$$
\int_{C} \omega^{\prime}=a^{\prime}+i b^{\prime}
$$




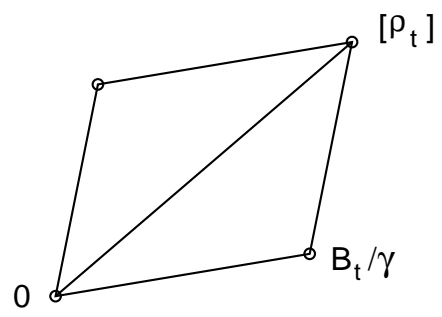

Figure 10. The content $C\left(\rho_{t}\right)$ is the full parallelogram when $t \in K \cup \infty$; otherwise, it is just the diagonal.

whenever $\int_{C} \omega=a+i b$. The isomorphism $H^{1}(X, \mathbb{R}) \cong \mathbb{C}^{2}$ given by

$$
[\sigma] \mapsto\left(\int \sigma \wedge \omega, \int \sigma \wedge \omega^{\prime}\right)
$$

sends $\left.H^{1}(X, \mathbb{Z})\right)$ to the points of the form $\left(a+i b, a^{\prime}+i b^{\prime}\right), a, b \in \mathcal{O}_{D}$. We note that $\omega$ and $\omega^{\prime}$ are eigenforms for real multiplication by $\mathcal{O}_{D}$ on $\operatorname{Jac}(X)$ [Mc1]. Clearly $B_{t}=A_{t} / \sqrt{a^{2}+b^{2}}$ satisfies

$$
\int B_{t} \wedge \omega=\int \rho_{t} \wedge \omega=\frac{a+i b}{\sqrt{a^{2}+b^{2}}} .
$$

This coordinate of $B_{t}$ simply moves around the unit circle in $\mathbb{C}$ as $t$ varies.

Graph of fluctuations. The more interesting quantity is

$$
z_{t}=\int B_{t} \wedge \omega^{\prime}=\frac{a^{\prime}+i b^{\prime}}{\sqrt{a^{2}+b^{2}}}
$$

The graph of the periodic function $\operatorname{Im} z_{t}$ is shown in Figure 3. The graph of $\operatorname{Re} z_{t}$ is identical, but shifted by $\pi / 2$. It is natural to define $z_{t}=0$ when $\mathcal{F}\left(\rho_{t}\right)$ is uniquely ergodic. Then $\left|z_{t}\right|$ is upper semicontinuous by Proposition 6.5 , and $\left|z_{t}\right| \rightarrow 0$ whenever $\tan (t) \rightarrow a \notin K$.

Triangle groups and continued fractions. Next we elaborate the notion of canonical fractions. Let $\Gamma=\operatorname{SL}(X, \omega)$ be the $(2,5, \infty)$ triangle group with generators (A.1). The group $\Gamma$ has only one cusp, whose orbit satisfies

$$
\Gamma \cdot \infty=\mathbb{P}^{1}(K)
$$

[Le1], [Mc2, §A]. This implies: 
Proposition A.2 Every $x \in \mathbb{P}^{1}(K)$ can be expressed uniquely as a ratio of relatively prime algebraic integers

$$
x=a / b, \quad a, b \in \mathcal{O}_{D},
$$

such that $\left(\begin{array}{ll}a & c \\ b & d\end{array}\right) \in \Gamma$ for some $c, d$, and $a x+b>0$.

(The last condition serves to fix the sign of $(a, b)$.)

We refer to the expression $x=a / b$ as a canonical fraction. Note that unique factorization in $\mathcal{O}_{D}$ only furnishes such ratios up to $(a, b) \sim \gamma^{2 n}(a, b)$. The group $\Gamma$ selects a distinguished representative from all such relative prime pairs.

The canonical fraction $x=a / b$ can be rapidly computed by a continuedfraction-type algorithm. The algorithm repeatedly translates by a multiple of $\gamma$ to arrange that $x \in[-\gamma / 2, \gamma / 2]$, and then replaces $x$ with $1 / x$ if $x \neq 0$.

Cylinders. The language of canonical fractions gives the following useful description of the cylinders on $(X, \omega)$.

Proposition A.3 Let $x=b / a$ be a canonical fraction in $K$. Then $(X, \omega)$ has two cylinders of slope $x$, whose core curves satisfy

$$
\int_{C_{1}} \omega=a+i b \text { and } \int_{C_{2}} \omega=\gamma(a+i b) .
$$

Proof. The statement holds for $x=0$, since $(X, \omega)$ has two horizontal closed geodesics of lengths 1 and $\gamma$ (as can be seen in Figure 9). For the general case, use the fact that all slopes in $K$ are equivalent under the action of $\operatorname{SL}(X, \omega)$.

Proof of Theorem A.1. First consider the case $t=0$. Then $(\sin t, \cos t)=$ $(a, b)=(0,1)$. As can be seen in Figure 9, the vertical foliation $\mathcal{F}\left(\rho_{0}\right)$ decomposes into cylinders of widths $\left(h_{1}, h_{2}\right)=\left(\gamma^{-1}, 1\right)$ whose core curves satisfy

$$
\int_{C_{1}} \omega=i=a+i b, \quad \int_{C_{2}} \omega=i \gamma .
$$

(Note that $h_{1}+h_{2}=\gamma$ gives the full width of $P$.) These curves are Poincaré dual to classes $\left[\alpha_{i}\right] \in H^{1}(X, \mathbb{Z})$ satisfying $\int \alpha_{i} \wedge \omega=\int_{C_{i}} \omega$ for $i=1,2$. In particular, $\left[\alpha_{1}\right]=A_{0}$.

From the cylinder decomposition

$$
\left[\rho_{0}\right]=h_{1}\left[\alpha_{1}\right]+h_{2}\left[\alpha_{2}\right],
$$


we see the vertices of $C\left(\rho_{0}\right)$ are given by

$$
\left\langle 0, h_{1}\left[\alpha_{1}\right], h_{2}\left[\alpha_{2}\right],\left[\rho_{0}\right]\right\rangle .
$$

Since $h_{1}\left[\alpha_{1}\right]=\gamma^{-1} A_{0}$ and $\sqrt{a^{2}+b^{2}}=1$, this together with (A.2) gives Theorem A.1 for $t=0$.

The other slopes follow similarly, using Proposition A.3 and the fact that the ratio of heights is always $\gamma$.

Radial limits of Teichmüller disk in $\mathbb{P} \mathcal{M} \mathcal{L}_{\boldsymbol{g}}$. To conclude we remark that the point $\left[\mathcal{F}\left(\rho_{t}\right)\right] \in \mathbb{P} \mathcal{M} \mathcal{L}_{g}$ represented by the measured foliation $\mathcal{F}\left(\rho_{t}\right)$ varies continuously as a function of $t$. At the same time, $\mathcal{F}\left(\rho_{t}\right)$ determines a Teichmüller geodesic ray which terminates at a well-defined lamination $\left[\lambda_{t}\right] \in \mathbb{P} \mathcal{M L}_{g}, g=2$. The union of these rays sweeps out a Teichmüller disk $f: \Delta \rightarrow \mathcal{T}_{g}$, with $\left[\lambda_{t}\right]$ as its radial limits. The behavior of these limits is well-known [Mas]:

1. If $\mathcal{F}\left(\rho_{t}\right)$ is uniquely ergodic, then $\left[\lambda_{t}\right]=\left[\mathcal{F}\left(\rho_{t}\right)\right]$.

2. If $\mathcal{F}\left(\rho_{t}\right)$ is periodic, and its cylinders have core curves $C_{1}, C_{2}$, then $\left[\lambda_{t}\right]=\left[C_{1}+C_{2}\right]$.

The function $t \mapsto\left[\lambda_{t}\right]$ is discontinuous precisely because in the second case, it forgets the heights of the 2 cylinders of $\mathcal{F}\left(\rho_{t}\right)$. In the case at hand, these heights have ratio $\gamma \neq 1$, so $\left[\lambda_{t}\right]$ is displaced from $\left[\mathcal{F}\left(\rho_{t}\right)\right]$ in the direction $\left[A_{t}\right]$. Thus Figure 3 , and its shift by $\pi / 2$, give the graphs of two coordinates of $\left[\lambda_{t}\right]$ with respect to a suitable charts on $\mathbb{P} \mathcal{M} \mathcal{L}_{g}$.

For related work on the Teichmüller curve of the regular octagon, see [SU].

\section{References}

[Ar1] P. Arnoux. Échanges d'intervalles et flots sur les surfaces. In Ergodic theory (Sem., Les Plans-sur-Bex, 1980), pages 5-38. Univ. Genéve, 1981.

[Ar2] P. Arnoux. Un exemple de semi-conjugaison entre un échange d'intervalles et une translation sur le tore. Bull. Soc. Math. France 116(1988), 489-500.

[ABB] P. Arnoux, J. Bernat, and X. Bressaud. Geometrical models for substitutions. Exp. Math. 20(2011), 97-127. 
[AS] P. Arnoux and T. A. Schmidt. Veech surfaces with non-periodic directions in the trace field. J. Mod. Dyn. 3(2009), 611-629.

[AY] P. Arnoux and J.-C. Yoccoz. Construction de diffeomorphisme pseudo-Anosov. C. R. Acad. Sci. Paris 292(1981), 75-78.

[BoS] Z. I. Borevich and I. R. Shafarevich. Number Theory. Academic Press, 1966.

[Bo] M. D. Boshernitzan. Rank two interval exchange transformations. Ergod. Th. \& Dynam. Sys. 8(1988), 379-394.

[Ca] E. Calabi. An intrinsic characterization of harmonic one-forms. In Global Analysis (Papers in Honor of K. Kodaira), pages 101-117. Univ. Tokyo Press, 1969.

[Dy] I. A. Dynnikov. Interval identification systems and plane sections of 3-periodic surfaces. Proc. Steklov Inst. Math. 263(2008), 65-77.

[Fa] A. Fathi. Some compact invariant sets for hyperbolic linear automorphisms of torii. Ergodic Theory Dynam. Systems 8(1988), 191-204.

[FLP] A. Fathi, F. Laudenbach, and V. Poénaru. Travaux de Thurston sur les surfaces. Astérisque, vol. 66-67, 1979.

[Fra] J. Franks. Anosov diffeomorphisms. In Global Analysis, Proc. Sympos. Pure Math., Vol. XIV, pages 61-93, 1970.

[Fr] D. Fried. The geometry of cross sections to flows. Topology 21(1982), 353-371.

[GH] P. Griffiths and J. Harris. Principles of Algebraic Geometry. Wiley Interscience, 1978.

[HLM] P. Hubert, E. Lanneau, and M. Möller. The Arnoux-Yoccoz Teichmüller disc. Geom. Funct. Anal. 18(2009), 1988-2016.

[Ken] R. Kenyon. The construction of self-similar tilings. Geom. Funct. Anal. 6(1996), 471-488.

[KS] R. Kenyon and J. Smillie. Billiards on rational-angled triangles. Comment. Math. Helv. 75(2000), 65-108.

[KSo] R. Kenyon and B. Solomyak. On the characterization of expansion maps for self-affine tilings. Discrete Comput. Geom. 43(2010), 577593. 
[KZ] M. Kontsevich and A. Zorich. Connected components of the moduli spaces of Abelian differentials with prescribed singularities. Invent. math. 153(2003), 631-678.

[Le1] A. Leutbecher. Über die Heckeschen Gruppen $G(\lambda)$. II. Math. Ann. 211(1974), 63-86.

[Le2] G. Levitt. Foliations and laminations on hyperbolic surfaces. Topology 22(1983), 119-135.

[LPV] J. H. Lowenstein, G. Poggiaspalla, and F. Vivaldi. Interval exchange transformations over algebraic number fields: the cubic ArnouxYoccoz model. Dyn. Syst. 22(2007), 73-106.

[Mas] H. Masur. Two boundaries of Teichmller space. Duke Math. J. 49(1982), 183-190.

[MT] H. Masur and S. Tabachnikov. Rational billiards and flat structures. In Handbook of Dynamical Systems, Vol. 1A, pages 1015-1089. North-Holland, 2002.

[MS] S. Mazurkiewicz and W. Sierpiǹski. Contribution à la topologie des ensembles denombrables. Fund. Math. 1(1920), 17-27.

[Mc1] C. McMullen. Billiards and Teichmüller curves on Hilbert modular surfaces. J. Amer. Math. Soc. 16(2003), 857-885.

[Mc2] C. McMullen. Teichmüller geodesics of infinite complexity. Acta Math. 191(2003), 191-223.

[Mc3] C. McMullen. Teichmüller curves in genus two: The decagon and beyond. J. reine angew. Math. 582(2005), 173-200.

[Mc4] C. McMullen. Prym varieties and Teichmüller curves. Duke Math. J. 133(2006), 569-590.

[Mc5] C. McMullen. Dynamics of $\mathrm{SL}_{2}(\mathbf{R})$ over moduli space in genus two. Annals of Math. 165(2007), 397-456.

[Mc6] C. McMullen. Foliations of Hilbert modular surfaces. Amer. J. Math. 129(2007), 183-215.

[Mc7] C. McMullen. Diophantine and ergodic foliations on surfaces. $J$. Topol. 6(2013), 349-360. 
[Mc8] C. McMullen. Navigating moduli space with complex twists. J. Eur. Math. Soc. 15(2013), 1223-1243.

[Mc9] C. McMullen. Moduli spaces of isoperiodic forms on Riemann surfaces. Preprint, 2012.

[Mo1] M. Möller. Variations of Hodge structures of a Teichmüller curve. J. Amer. Math. Soc. 19(2006), 327-344.

[Mo2] M. Möller. Affine groups of flat surfaces. In A. Papadopoulos, editor, Handbook of Teichmüller Theory, volume II, pages 369-387. Eur. Math. Soc., 2009.

[Nov] S. P. Novikov. The Hamiltonian formalism and a multivalued analogue of Morse theory. Russian Math. Surveys 37(1982), 1-56.

[Sch] S. Schwartzman. Asymptotic cycles. Ann. of Math. 66(1957), 270284.

[SU] J. Smillie and C. Ulcigrai. Geodesic flow on the Teichmüller disk of the regular octagon, cutting sequences and octagon continued fractions maps. Preprint, 2010.

[Str] K. Strebel. Quadratic Differentials. Springer-Verlag, 1984.

[Sul] D. Sullivan. Cycles for the dynamical study of foliated manifolds and complex manifolds. Invent. math. 36(1976), 225-255.

[Th] W. P. Thurston. On the geometry and dynamics of diffeomorphisms of surfaces. Bull. Amer. Math. Soc. 19(1988), 417-431.

[V1] W. Veech. The metric theory of interval exchange transformations. III. The Sah-Arnoux-Fathi invariant. Amer. J. Math. 106(1984), 1389-1422.

[V2] W. Veech. Teichmüller curves in moduli space, Eisenstein series and an application to triangular billiards. Invent. math. 97(1989), $553-583$.

[V3] W. A. Veech. Strict ergodicity in zero dimensional dynamical systems and the Kronecker-Weyl theorem mod 2. Trans. Amer. Math. Soc. 140(1969), 1-33. 
[Z] A. Zorich. How do the leaves of a closed 1-form wind around a surface? In Pseudoperiodic Topology, Amer. Math. Soc. Transl. Ser. 2, pages 135-178. Amer. Math. Soc., 1999.

Mathematics Department

HARVARD UNIVERSITY

1 OXFORD ST

Cambridge, MA 02138-2901 